\title{
Multi-objective Ant Colony Optimization for Requirements Selection
}

\author{
José del Sagrado • Isabel M. del Águila • \\ Francisco J. Orellana
}

Received: date / Accepted: date

\begin{abstract}
The selection of a set of requirements between all the requirements previously defined by customers is an important process, repeated at the beginning of each development step when an incremental or agile software development approach is adopted. The set of selected requirements will be developed during the actual iteration. This selection problem can be reformulated as a search problem, allowing its treatment with metaheuristic optimization techniques. This paper studies how to apply Ant Colony Optimization algorithms to select requirements. First, we describe this problem formally extending an earlier version of the problem, and introduce a method based on Ant Colony System to find a variety of efficient solutions. The performance achieved by the Ant Colony System is compared with that of Greedy Randomized Adaptive Search Procedure and Non-dominated Sorting Genetic Algorithm, by means of computational experiments carried out on two instances of the problem constructed from data provided by the experts.
\end{abstract}

Keywords Software requirements · Search based Software Engineering · Ant colony optimization · Next release problem

\section{Introduction}

Software development organizations fail many times to deliver its products within schedule and budget. Statistical studies, and CHAOS Reports (Johnson, 2003) published since 1994, reveal that, frequently, tasks related to requirements lead software project to the disaster. As Kotonya and Sommerville

Departamento de Informática. Universidad de Almería, Ctra. Sacramento s/n, 04120 Almería, Spain

first address

Tel.: +34-950015086

Fax: +34-950015129

E-mail: jsagrado@ual.es 
(1998) suggest, one of the major problems we face when developing large and complex software systems is the one related with requirements. The concept of requirement, in its broadest sense, must be understood as a logical unit of behavior that is specified by including functional and quality aspects; other authors as Ruhe and Saliu (2005) use instead the concept of feature. Stakeholders propose some desired functionalities that software managers must filter in order to define the set of requirements to include in the final software product.

Usually, during the lifetime of a software product, we are faced with the problem of selecting a subset of requirements from the whole set of candidate requirements. Enhancements to include into the next software release cannot be randomly selected since there are many factors involved. Within this scenario, customers demand their own software enhancements, but all of them cannot be included in the software product, mainly due to the existence limited resources (e.g. availability of man-month in a given software project). In most cases, it is not feasible to develop all the new functionalities suggested. Hence each new feature competes against each other to be included in the next release.

This task of selecting a set of requirements, which until now only appeared when defining new versions of widely distributed software products, becomes important within the incremental approaches of software development, and specially in the agile approaches. Agile methods promote a disciplined project management process that encourages frequent inspection and adaption. These software development methodologies are based on iterative development, advocating for frequent "releases" in short development cycles, called timeboxes, in order to improve productivity and introduce checkpoints. Each iteration works through a full cycle, generating a software release that has to be shown to stakeholders. These approaches focus on the quick adaptation of software to the changing realities.

Within this prospective, the challenge of Software Engineering consists on defining specific techniques or methods that improve the way requirements are selected. The problem of choosing a set of requirements fulfilling certain criteria, such as minimal cost or maximal client satisfaction, is a good candidate for the application of metaheuristics. Specifically, this paper shows how Ant Colony Optimization (ACO) systems can be applied to problems of requirements selection. Our solution offers to developers and stakeholders a set of possibilities satisfying several objectives, i.e. the Pareto front. The idea is to help people to take a decision about which set of requirements has to be included in the next release during the software development applying both, agile or classical software development approaches. The proposed ACO system is evaluated by means of a compared analysis with Non-dominated Sorting Genetic Algorithm (NSGA-II) (Deb, 2001; Deb et al, 2002) and Greedy Randomized Adaptive Search Procedure (GRASP) (Feo and Resende, 1989; Pitsoulis and Resende, 2003; Resende and Ribeiro, 2003); both adapted to the problem of requirements selection.

The rest of the paper is organized in six sections. Section 2 summarizes the basic Requirements Engineering concepts, focusing in requirement selec- 
tion process for the next software release, together with the description and definition of the problem of selection of requirements to be included in the next software release, know as Next Release Problem (NRP) (Bagnall et al, 2001). Section 3 describes the metaheuristic technique applied in this work, Ant Colony Optimization. Specifically, we focus on its application in multiobjective optimization problems. Section 4 is devoted to the study of how multi-objective ACO can be used to find a set of non-dominated solutions to NRP. In Section 5, the experimental evaluation is carried out by comparing ACO with GRASP and NSGA-II approaches. The analysis of the results is presented in Section 6. Section 7 presents related work. Finally, in Section 8 we give some conclusions and the future works that can extend this study.

\section{Requirements Selection}

Requirements related tasks are inherently difficult Software Engineering activities. Descriptions of requirements are supposedly written in terms of the domain, describing how this environment is going to be affected by the new software product. In contrast, other software processes and artifacts are written in terms of the internal software entities and properties (Cheng and Atlee, 2007).

The problem of selecting the subset of requirements among a whole set of candidate requirements proposed by a group of customers is not a straightforward problem, since there are many factors involved. Customers, seeking their own interest, demand the set of enhancements they consider important, but not all customer needs can be satisfied. On the one hand, each requirement means a cost in effort terms that the company must assume, but company resources are limited. On the other hand, neither all the customers are equally important for the company, nor the requirements are equally important for the customers. Market factors can also drive this selection process; the company may be interested on satisfying the newest customers needs, or they may consider desirable to guarantee that every customer sees fulfilled at least one of their proposed requirements. Also, requirements show interactions that impose a certain development order or either conflicts between them, limiting the alternatives to be chosen.

During the software development process many interaction types between two or more requirements can be identified. Karlsson et al (1997) were the first in proposing a list of interaction types. Later, Carlshamre et al (2001) propose a set of interaction types as result of an in-depth study of interactions in distinct sets of requirements coming from different software development projects. Although interaction types are semantically different, in practice they can be grouped into:

- Implication or precedence. $r_{i} \Rightarrow r_{j}$. A requirement $r_{i}$ cannot be selected if a requirement $r_{j}$ has not been implemented yet.

- Combination or coupling. $r_{i} \otimes r_{j}$. A requirement $r_{i}$ cannot be included separately from a requirement $r_{j}$. 
- Exclusion. $r_{i} \oplus r_{j}$. A requirement $r_{i}$ can not be included together with a requirement $r_{j}$.

- Revenue-based. The development of a requirement $r_{i}$ implies that some others requirements will increase or decrease their value.

- Cost-based. The development of a requirement $r_{i}$ implies that some others requirements will increase or decrease their implementation cost.

These interactions must be considered as constraints in the requirements selection problem. So, two main goals are usually considered: maximize the customers satisfaction and minimize the required software development effort satisfying the given constraints. Therefore, optimization techniques can be used to find optimal or near optimal solutions in a reasonable amount of time. Our aim is to define the requirements selection problem as a science (Ruhe and Saliu, 2005), formalizing the problem and applying computational algorithms to generate good solutions.

\subsection{Previous and Related Works}

The Search-Based Software Engineering (SBSE) area is the research field which proposes the application of search-based optimization algorithms to tackle problems in Software Engineering (Harman and Jones, 2001; ?). In this section we provide a comprehensive review of different approaches that can be found in the literature to tackle with the requirements selection problem (an earlier review can be found in del Sagrado et al (2010b)).

As a problem in which is necessary to evaluate multiple conflicting objectives, its solution requires to find the best compromise between the different objectives. In order to achieve this, we can proceed in two ways. The first approach consists in transforming the multi-objective problem into a single objective problem. To do that, we need to combine the different objectives into the single one by means of an aggregation function (e.g. a weighted sum or product). This is the approach chosen by Bagnall et al (2001), they formulate the problem of selecting a subset of requirements (i.e. Next Release Problem-NRP) having as goal meet the customer's needs, minimizing development effort and maximizing customers satisfaction and apply hill climbing, greedy algorithms and simulated annealing. Later, Baker et al (2006) demonstrate that these metaheuristics techniques can be applied to a real-world NRP out-performing expert judgment. Greer and Ruhe (2004) study the generation of feasible assignments of requirements to increments taking into account different stakeholders perspectives and resources constraints. The optimization method they used is iterative and essentially based on a genetic algorithm.

With respect to the application of ACO to tackle the single-objective version of NRP, the first approach can be found in the works of del Sagrado and del Águila (2009) and del Sagrado et al (2010a) where an Ant Colony System (ACS) is proposed. Jiang et al (2010) incorporate into ACO a local search for improving the quality of solution found. Nontheless, all of these approaches do not take into account the existence of interactions between requirements. 
The problem of requirements selection, including interactions between requirements, is introduced in the works of del Sagrado et al (2011) and de Souza et al (2011) by adapting the ACS algorithm.

The second approach to multi-objective optimization builds a set with all the solutions found that are not dominated by any other (this is known as the set of efficient solutions or Pareto-optimal set). The decision maker will select a solution from this set according to personal criteria. The works of Saliu and Ruhe (2007), Zhang et al (2007), Finkelstein et al (2008, 2009) and Durillo et al (2009), study the NRP problem from the multi-objective point of view, either as an interplay between requirements and implementation constraints (Saliu and Ruhe, 2007) or considering multiple objectives as cost-value (Zhang et al, 2007) or different measures of fairness (Finkelstein et al, 2008, 2009), or applying several algorithms based on genetic inspiration as NSGA-II, MOCell and PAES (Durillo et al, 2009, 2011). However, to date, no ACO approach has been applied to multi-objective NRP.

It is worth to note that having more than one valid solution, as in the multi-objective approach, constitute a valuable aid for experts who must decide what is the set of requirements that has to be considered in the next software release. Requirements managers analyze these alternatives and their data (e.g. number of customer covered, additional information about risky requirements), before selecting a solution (i.e. the set of requirements to be developed) according business strategies. Thus, it is considerably helpful, for any software developer, to have these techniques available either embedded in a CASE (Computer-Aided Software Engineering) tool (del Sagrado et al, 2012), or within a deccision support tool for release planning (Carlshamre, 2002; Momoh and Ruhe, 2006).

\subsection{NRP Formulation}

Let $\mathbf{R}=\left\{r_{1}, r_{2}, \ldots, r_{n}\right\}$ be the set of requirements that are still to be implemented. These requirements represent enhancements to the current system that are suggested by a set of $m$ customers and are candidates to be included in the next software release. Customers are not equally important. So, each customer $i$ will have an associated weight $w_{i}$, which measures its importance. Let $\mathbf{W}=\left\{w_{1}, w_{2}, \ldots, w_{m}\right\}$ be the set of customers weights.

Each requirement $r_{j}$ in $\mathbf{R}$ has an associated development $\operatorname{cost} e_{j}$, which represents the effort needed in its development. Let $\mathbf{E}=\left\{e_{1}, e_{2}, \ldots, e_{n}\right\}$ be the set of requirements efforts. On many occasions, the same requirement is suggested by several customers. However, its importance or priority may be different for each customer. Thus, the importance that a requirement $r_{j}$ has for customer $i$ is given by a value $v_{i j}$. The higher the $v_{i j}$ value, the higher is the priority of the requirement $r_{j}$ for customer $i$. A zero value for $v_{i j}$ represents that customer $i$ has not suggested requirement $r_{j}$. All these importance values $v_{i j}$ can be arranged under the form of an $m \times n$ matrix. 


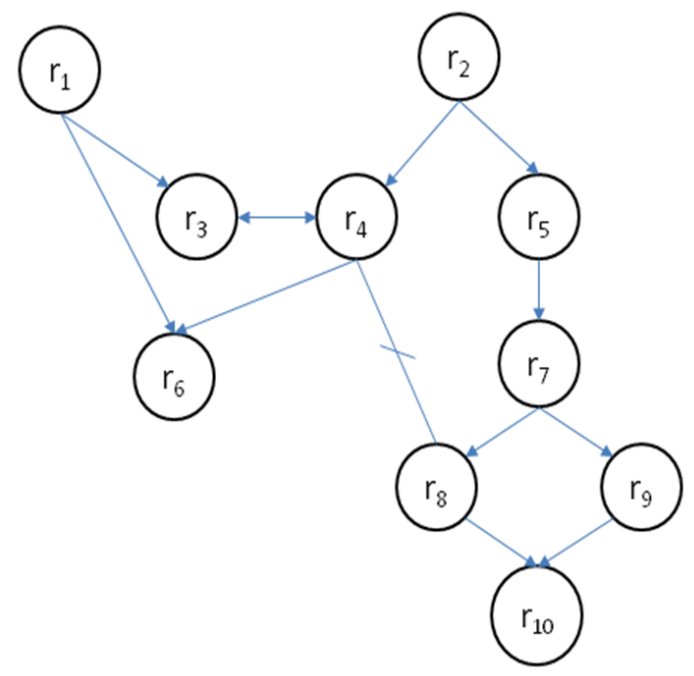

Fig. 1 Functional interactions represented as a graph $G=(\mathbf{R}, \mathbf{I}, \mathbf{J}, \mathbf{X})$.

The global satisfaction, $s_{j}$, or the added value given by the inclusion of a requirement $r_{j}$ in the next software release, is measured as a weighted sum of its importance values for all the customers and can be formalized as:

$$
\mathrm{s}_{j}=\sum_{i=1}^{m} w_{i} * v_{i j},
$$

The set of requirements satisfaction computed in this way is denoted as $\mathbf{S}=\left\{s_{1}, s_{2}, \ldots, s_{n}\right\}$. Requirements interactions can be divided into two groups. The first consists of the functional interactions: implication, combination and exclusion. The second one includes those interactions that imply changes in the amount of resources needed or the benefit related to each requirement: revenuebased and cost-based. Functional interactions can be explicitly represented as a graph $G=(\mathbf{R}, \mathbf{I}, \mathbf{J}, \mathbf{X})$ where:

- $\mathbf{R}$ (the set of requirements) is the set of nodes

- $\mathbf{I}=\left\{\left(r_{i}, r_{j}\right) \mid r_{i} \Rightarrow r_{j}\right\}$ each pair $\left(r_{i}, r_{j}\right) \in \mathbf{I}$ is an implication interaction and will be represented as a directed link $r_{i} \rightarrow r_{j}$

$-\mathbf{J}=\left\{\left(r_{i}, r_{j}\right) \mid r_{i} \oplus r_{j}\right\}$ each pair $\left(r_{i}, r_{j}\right) \in \mathbf{J}$ is a combination interaction and will be represented as a double directed link $r_{i} \leftrightarrow r_{j}$

- $\mathbf{X}=\left\{\left(r_{i}, r_{j}\right) \mid r_{i} \otimes r_{j}\right\}$ each pair $\left(r_{i}, r_{j}\right) \in \mathbf{X}$ is an exclusion interaction and will be represented as a crossed undirected link

For example, consider the set of requirements $\mathbf{R}=\left\{r_{1}, r_{2}, \ldots, r_{10}\right\}$ and the following functional dependencies $\mathbf{I}=\left\{\left(r_{1}, r_{3}\right),\left(r_{1}, r_{6}\right),\left(r_{2}, r_{4}\right),\left(r_{2}, r_{5}\right),\left(r_{4}, r_{6}\right)\right.$, $\left.\left(r_{5}, r_{7}\right),\left(r_{7}, r_{8}\right),\left(r_{7}, r_{9}\right),\left(r_{8}, r_{10}\right),\left(r_{9}, r_{10}\right)\right\}, \mathbf{J}=\left\{\left(r_{3}, r_{4}\right)\right\}, \mathbf{X}=\left\{\left(r_{4}, r_{8}\right)\right\}$. We can represent all of these sets as the graph showed in Figure 1. 
The second group of interactions represents changes in satisfaction and effort values of individual requirements. These two interaction types can be modelled as a pair of $n \times n$ simetric diagonal matrices:

- $\Delta S$ where each element $\Delta s_{i j}$ of this matrix represents the increment or decrement of $s_{j}$ when $r_{i}$ and $r_{j}$ are implemented in the same release,

$-\Delta E$ where each element $\Delta e_{i j}$ of this matrix represents the increment or decrement of $e_{j}$ when $r_{i}$ and $r_{j}$ are implemented simultaneously,

in which the elements in the diagonal are equal to zero.

In order to define the next software release, we have to select a subset of requirements $\hat{\mathbf{R}}$ included in $\mathbf{R}$, which maximize satisfaction and minimize development effort. The satisfaction and development effort of the next release can be obtained, respectively, as:

$$
\begin{aligned}
\operatorname{sat}(\hat{\mathbf{R}}) & =\sum_{j \in \hat{\mathbf{R}}} s_{j} \\
\operatorname{eff}(\hat{\mathbf{R}}) & =\sum_{j \in \hat{\mathbf{R}}} e_{j}
\end{aligned}
$$

where $j$ is an abbreviation for requirement $r_{j}$. For a given release there is a cost bound $B$, that cannot be overrun. Under this set of circumstances the requirements selection problem for the next software release can be formulated as an optimization problem:

$$
\begin{aligned}
& \text { maximize } \operatorname{sat}(\hat{\mathbf{R}}) \\
& \text { minimize } \operatorname{eff}(\hat{\mathbf{R}})
\end{aligned}
$$

subject to the restriction $\sum_{j \in \hat{\mathbf{R}}} e_{j} \leq B$ due to the particular effort bound $B$ applied, where $\hat{\mathbf{R}}$ also fulfills functional requirements interactions. Thus, two conflicting objectives, such as maximizing customer satisfaction and minimizing software development effort, are optimized at the same time within a given software development effort bound. The solution to this problem consists of a set of solutions known as Pareto-optimal set (Coello et al, 2007; Deb, 2001; Srinivas and Deb, 1994).

\subsection{Basic Instance of NRP}

Bagnall et al (2001) defines a basic NRP as a NRP where no requirement has any prerequisites. So, following Bagnall formulation, any NRP can be transformed into a basic NRP simply by grouping together each requirement and its ancestors in the graph of precedence interactions (i.e. requirements for which there is a path in the graph ending in the requirement that is being considered). However, there are also other types of interactions besides implication (i.e. combination, exclusion, revenue-based and cost-based interactions) that also have to be taken into account when solving a NRP. Therefore, we are going 
to extend the work of Bagnall et al (2001), defining a process for transforming a NRP into a basic NRP, before applying meta-heuristics optimization techniques.

The process to transform a NRP into a basic NRP is made on three steps:

1. Each pair $\left(r_{i}, r_{j}\right) \in \mathbf{J}$ is transformed into a new requirement $r_{i+j}$, with $s_{i+j}=s_{i}+s_{j}$ and $e_{i+j}=e_{i}+e_{j}$. This produces a new functional interactions graph $G^{\prime}=(\mathbf{R}, \mathbf{I}, \mathbf{J}, \mathbf{X})$ in which $\mathbf{J}=\emptyset$.

2. A pair $\left(r_{i}, r_{j}\right) \in \mathbf{X}$ requires the alternative deletion of each requirement together with its descendants from $G^{\prime}$ (i.e. requirements for which there is a path in the graph starting in the requirement that is being considered), resulting two new functional interactions graphs $G_{i}^{\prime}$ and $G_{j}^{\prime}$. This process is repeated from these new graphs until there are no exclusion interactions.

3. For each interactions graph $G_{i}^{\prime}$ obtained in step 2, a basic NRP is builded by grouping together implication interactions (i.e. each requirement and its ancestors in the graph) $r_{j}^{+}=\left\{r_{j}\right\} \cup \operatorname{ancestors}\left(r_{j}\right)$.

Revenue-based and cost-based interactions are updated taking into account these graphs and the values of the original NRP:

1. Each pair $\left(r_{i}, r_{j}\right) \in \mathbf{J}$ implies that, starting from $\Delta S^{\prime}=\Delta S, \Delta s_{i k}^{\prime}=$ $\Delta s_{i k}+\Delta s_{j k}$ and $\Delta s_{k i}^{\prime}=\Delta s_{i k}^{\prime}$, for $i \neq k$, and $\Delta s_{i i}^{\prime}=0$. After that, the row and column associated to $r_{j}$ are deleted from $\Delta S^{\prime}$. The same process applies to cost-based interactions. As result, we obtain new revenue-based, $\Delta S^{\prime}$, and cost-based, $\Delta E^{\prime}$, matrices.

2. A pair $\left(r_{i}, r_{j}\right) \in \mathbf{X}$ requires the alternative deletion of the rows and columns associated to each requirement and its descendants in $G^{\prime}$ from $\Delta S$ and $\Delta E$. Thus, the deletion of the rows and columns associated to $\left\{r_{i}\right\} \cup$ descendants $\left(r_{i}\right)$ produces $\Delta S_{i}^{\prime}$ and $\Delta E_{i}^{\prime}$, whereas that of $\left\{r_{j}\right\} \cup$ descendants $\left(r_{j}\right)$ produces $\Delta S_{j}^{\prime}$ and $\Delta E_{j}^{\prime}$. This process is repeated from these new matrices and the graphs $G_{i}^{\prime}$ and $G_{j}^{\prime}$ until there are no exclusion interactions.

3. For each $r_{j}^{+}$in the basic NRP builded from an interactions graph $G_{i}^{\prime}$ together with its pair of associated matrices $\Delta S_{i}^{\prime}$ and $\Delta E_{i}^{\prime}$, we define revenuebased, $\Delta S^{+}$, and cost-based, $\Delta E^{+}$, matrices as:

$$
\begin{aligned}
& \Delta s_{i j}^{+}= \begin{cases}\sum_{r_{k} \in r_{i}^{+} \backslash r_{i}^{+} \cap r_{j}^{+}} \Delta s_{k l}^{\prime}, & \text { if } i \neq j, \\
r_{l} \in r_{j}^{+} \backslash r_{i}^{+} \cap r_{j}^{+} & \text {if } i=j, \\
0, & \end{cases} \\
& \Delta e_{i j}^{+}= \begin{cases}\sum_{r_{k} \in r_{i}^{+} \backslash r_{i}^{+} \cap r_{j}^{+}} \Delta e_{k l}^{\prime}, & \text { if } i \neq j, \\
r_{l} \in r_{j}^{+} \backslash r_{i}^{+} \cap r_{j}^{+} & \text {if } i=j . \\
0, & \end{cases}
\end{aligned}
$$

For example, if we consider the NRP depicted in Figure 1 as a functional interactions graph, then during the first stage of the process, combination interactions are erased and the graph shown in Figure 2 (a) is obtained. The 


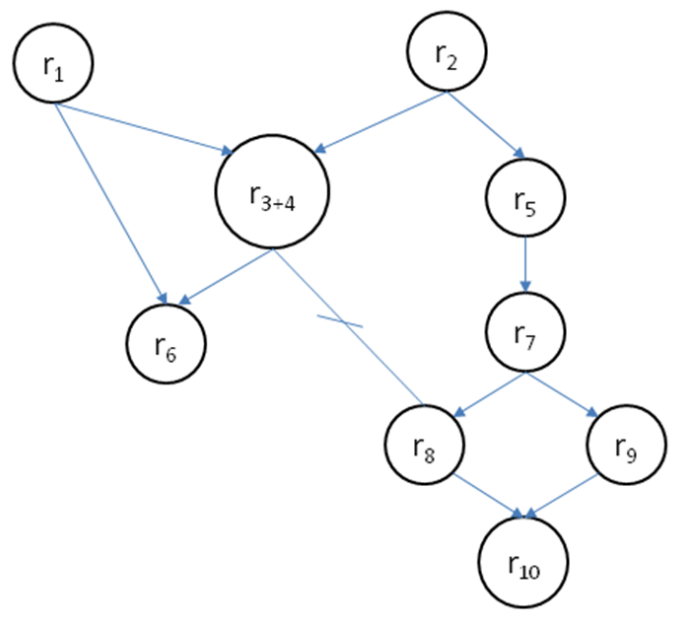

a) Functional interactions graph $G^{\prime}$ after eliminating combination interactions
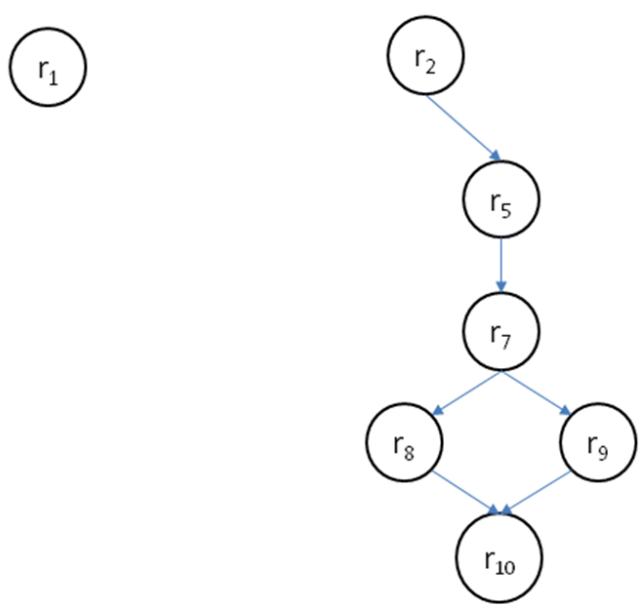

b) Functional interactions graph $G_{3+4}^{\prime}$ after deleting $r_{3+4}$ and its descendants from $G^{\prime}$

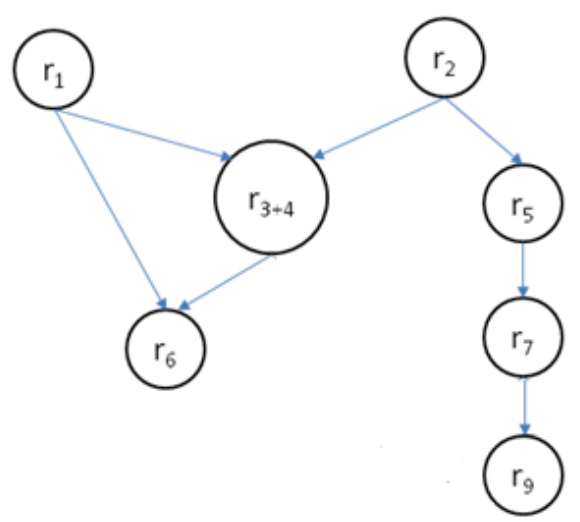

c) Functional interactions graph $G_{8}^{\prime}$ after deleting $r_{8}$ and its descendants from $G^{\prime}$

Fig. 2 Transformation of a NRP into a basic NRP 
second stage takes this functional interactions graph and proceeds to eliminate exclusion dependences. Thus, requirement $r_{3+4}$ and its descendants are deleted which produces the graph $G_{3+4}^{\prime}$ (see Figure 2(b)) and the elimination of requirement $r_{8}$ along with its descendants, produces the graph $G_{8}^{\prime}$ (see Figure $2(\mathrm{c})$ ). Last two graphs contain only implication interactions and define the basic NRP.

Once we get the set of functional interactions graphs, containing only implication interactions, the transformation ends simply by grouping together each requirement and its ancestors in each graph, obtaining several basic NRPs. For example, if we consider the graph $G_{3+4}^{\prime}$ in Figure 2(b) we get $r_{1}^{+}=\left\{r_{1}\right\}, r_{2}^{+}=\left\{r_{2}\right\}, r_{5}^{+}=\left\{r_{2}, r_{5}\right\}, r_{7}^{+}=\left\{r_{2}, r_{5}, r_{7}\right\}, r_{8}^{+}=\left\{r_{2}, r_{5}, r_{7}, r_{8}\right\}$, $r_{9}^{+}=\left\{r_{2}, r_{5}, r_{7}, r_{9}\right\}$ and $r_{10}^{+}=\left\{r_{2}, r_{5}, r_{7}, r_{8}, r_{9}, r_{10}\right\}$ where $r_{i}^{+}$denotes the set of requirements $\left\{r_{i}\right\} \cup$ ancestors $\left(r_{i}\right)$.

After executing these processes, we obtain a set of graphs, containing only implication interactions that can be treated applying the Bagnall's approach. Observe that the presenece of exclusion interactions causes an alternative consideration of requirements and the appearance of a greater number of interactions graphs. This is due to the inner nature of exclusion. If there is an exclusion interaction between two requirements, $\left(r_{i}, r_{j}\right) \in \mathbf{X}$, by definition these requirements are incompatible software features tnat can not be included in the same software product at the same time. Requirements Engineering faces this problem as a negotiation problem. Project developers must obtain and agreement by the elimination of one of them (i.e. discarding one of the requirements) or by generating two different software applications. For example, consider the exclusive requirements $r_{i}$ : "all users should be able to search for data about both products and customers" and $r_{j}$ : "only personnel with a high security level should be able to search for customers classified as military related". Each one of them leads to a different software product and we need to perform different search processes defining different alternatives for the next software release.

Note that a more restrictive special case arises when requirements are basic and independent: for all $r_{i} \in \mathbf{R}, r_{i}^{+}=\left\{r_{i}\right\}$. Bagnall et al (2001) has shown that NRP is an instance of $0 / 1$ knapsack problem, which is NP-hard. This result means that large instances of the problem cannot be solved by exact optimization techniques in a polynomial time. Nonetheless, in this situation the use of metaheuristics is suitable because they can find near-optimal solutions to NRP spending a reasonable amount of time.

\section{Multi-objective Ant Colony Optimization for the NRP}

Ant Colony Optimization (ACO) has been applied to multi-objective optimization problems (Iredi et al, 2001; Doerner et al, 2004; Häckel et al, 2008) using a multi-colony strategy, extending the Ant Colony System (ACS) (Dorigo et al, 2006; Dorigo and Stützle, 2004). Iredi et al (2001) and Häckel et al (2008) propose a multi-colony method to solve multi-objective optimization problems 
when the objectives cannot be ordered by importance, in which each colony searches for a solution in a different region of the Pareto-front. Whereas Doerner et al (2004) propose the use of a single colony in which each ant searches in a different direction of the Pareto front, and apply it specifically to a portfolio optimization problem. This last approach enables us to extend in a natural way the ACS for NRP, described in (del Sagrado et al, 2010a, 2011), to the multi-objective case by searching in different directions on the Pareto front.

In ACO algorithms, each ant builds its its solution from an initial node (requirement) which is selected randomly. At each stage, an ant locates a set of neighboring nodes to visit (these requirements must satisfy the restrictions of the problem). Among all of them it selects one in a probabilistic way, taking into account the pheromone level and heuristic information. The level of pheromone deposited in an arc from node $r_{i}$ to node $r_{j}, \tau_{i j}$, is stored in a matrix $\tau$, whereas the heusirstic information about the problem is represented as the value $\eta_{i j}$ and have to be defined based on the problem itself.

Boehm et al (2001) have proposed several metrics for software products. These metrics help in the assessment of the quality of a software product when its development has finished and also in the estimation of the effort of a new software project. One such metrics is the productivity of development teams, conceived as the number of developed units (thousands of lines of code, function points) per unit of effort (man-months). Since we have to select the a set of requirements, we should consider a metric of this type, measuring the productivity in terms of the customers' benefit. This concept has been applied in the selection and triage of the requirements (Davis, 2003; Simmons, 2004). In NRP we can define a productivity metric associated to a requirement $r_{j}$, as, $s_{j} / e_{j}$ which is the level of satisfaction obtained by the customers when including this requirement in an increment based on the effort applied in its development. Hence, we define:

$$
\eta_{i j}=\xi \frac{s_{j}}{e_{j}}
$$

where $\xi$ is a normalization constant.

Let $\mathbf{R}^{k} \subseteq \mathbf{R}$ be the partial solution to the problem built by ant $k$ and assume that the ant is located at node $r_{i}$, then

$$
\begin{aligned}
N_{i}^{k}=\left\{r_{j} \mid r_{j} \notin \mathbf{R}^{\mathbf{k}},\right. & \operatorname{eff}\left(\mathbf{R}^{\mathbf{k}}\right)+e_{j} \leq B, \\
& \left.\mathbf{R}^{\mathbf{k}} \cup\left\{r_{j}\right\} \text { fullfills functional interactions }\right\}
\end{aligned}
$$

represents the set of non visited neighbors nodes that can be reached by ant $k$ from node $r_{i}$. That is, for ant $k$ a node $r_{j}$ is visible from node $r_{i}$ if and only if $r_{j}$ has not been previously visited, its inclusion in the partial solution $\mathbf{R}^{\mathbf{k}}$ does not exceed the fixed development effort bound $B$ and does not break functional interactions.

In the multi-objective $\mathrm{ACO}$, there will be a pheromone matrix $\tau^{g}$ for each one of the objectives, $g \in \mathbf{O}$ (for NRP the set of objectives is $\mathbf{O}=\{s, e\}$, where $s$ represents satisfaction and $e$ represents effort) and the solution constructed 
by an ant is based on a weighted combination of these pheromone matrices. The weights $\lambda_{g}^{k}$, that ant $k$ assigns to the different objectives, measure the relative importance of the optimization criteria and should be distributed uniformly over the different regions of the Pareto front. For the NRP case, if the colony has $z$ ants, then the weights for users' satisfaction and development effort used by an ant $k \in[0, z]$ are defined respectively as $\lambda_{s}^{k}=k /(z-1)$ and $\lambda_{e}^{k}=1-\lambda_{s}^{k}$. Note that weights are kept fixed over the ant's lifetime (i.e. time expended by the ant to build its solution).

In the ACS algorithm, each ant builds, in a progressive way, a solution to the problem. During the construction process of the solution, ant $k$ selects from node $r_{i}$ the next node $r_{j}$ to visit applying a pseudorandom proportional rule (Dorigo and Gambardella, 1997) that takes into account the weights $\lambda_{g}^{k}$ (Doerner et al, 2004):

$$
j=\left\{\begin{array}{l}
\operatorname{argmax}_{u \in N_{i}^{k}}\left\{\left[\sum_{g \in \mathbf{O}} \lambda_{g}^{k} \tau_{i u}^{g}\right]^{\alpha}\left[\eta_{i u}\right]^{\beta}\right\}, \text { if } q \leq q_{0}, \\
\text { otherwise. }
\end{array}\right.
$$

where $q$ is a random number uniformly distributed in $[0,1], q_{0} \in[0,1]$ is a parameter that determines a trade-off between exploitation $\left(q \leq q_{0}\right)$ and exploration, and $u \in N_{i}^{k}$ is a node randomly selected. An ant $k$, selects randomly from node $r_{i}$ the next node $r_{j}$ to visit with a probability $p_{i j}^{k}$ given by (Doerner et al, 2004):

$$
p_{i j}^{k}= \begin{cases}\frac{\left[\sum_{g \in \mathbf{O}} \lambda_{g}^{k} \tau_{i j}^{g}\right]^{\alpha}\left[\eta_{i j}\right]^{\beta}}{\sum_{h \in N_{i}^{k}}\left[\sum_{g \in \mathbf{O}} \lambda_{g}^{k} \tau_{i h}^{g}\right]^{\alpha}\left[\eta_{i h}\right]^{\beta}}, & \text { if } j \in N_{i}^{k}, \\ 0, & \text { otherwise. }\end{cases}
$$

where the parameters $\alpha$ and $\beta$ reflect the relative influence of the pheromone with respect to the heuristic information. For example, if $\alpha=0$ the nodes with higher heuristic information values will have a higher probability of being selected (the ACO algorithm will be close to a classical greedy algorithm). If $\beta=0$ the nodes with higher pheromone value will be preferred in order to be selected. From these two examples, it is easy to deduce that is needed a balance between heuristic information and pheromone level.

While building its solution each ant $k$ in the colony updates pheromone locally. If it chooses the transition from node $r_{i}$ to $r_{j}$, then it has to update the pheromone level of the corresponding arc for each objective $g$ applying the following rule:

$$
\tau_{i j}^{g}=(1-\varphi) * \tau_{i j}^{g}+\varphi \tau_{0}
$$

where $\varphi \in[0,1]$ is the pheromone decay coefficient and $\tau_{0}$ is the initial pheromone value, which is defined as $\tau_{0}=1 /|\mathbf{R}|$. Each time an arc is visited, its pheromone level decreases making it less attractive for subsequent 


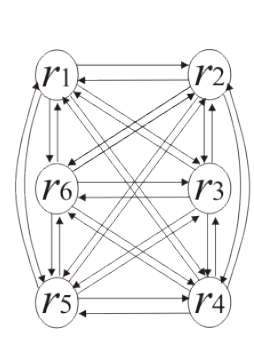

( a )

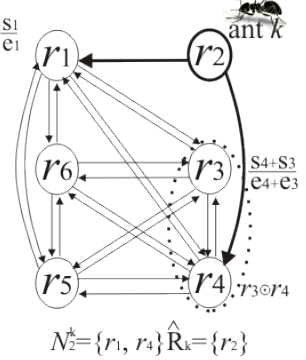

(b)

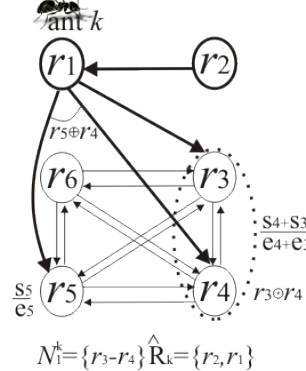

(c)

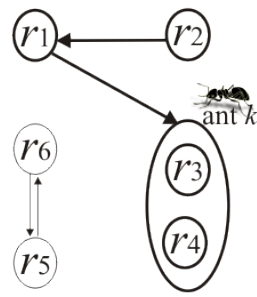

$N_{3-4}^{k}=\{\} \hat{R}_{k}=\left\{r_{2}, r_{1}, r_{3}-r_{4}\right\}$

(d)

Fig. 3 Search process in ACS.

ants. Thus, this local update process encourages the exploration of other arcs avoiding premature convergence.

Once all ants in the colony have built a solution, only the two ants that have obtained the best solutions reinforce pheromone on the arcs that are part of the best solutions. They update pheromone globally for each objective $g \in \mathbf{O}$ and each arc, $(i, j)$, included in the best solutions applying the following rule:

$$
\tau_{i j}^{g}=(1-\rho) * \tau_{i j}^{g}+\rho \Delta \tau_{i j}^{g}
$$

where $\rho \in[0,1]$ is the pheromone evaporation rate and $\Delta \tau_{i j}^{g}$ represents the increase of pheromone with respect to objective $g$.

In the NRP case, we have two objectives, $\mathbf{O}=\{s, e\}$, satisfaction and effort, so ,for a given best solution $\hat{\mathbf{R}}$, we define two pheromone increments ( $\Delta \tau_{i j}^{s}$ for satisfaction and $\Delta \tau_{i j}^{e}$ for effort) as:

$$
\begin{aligned}
\Delta \tau_{i j}^{s} & =\frac{1}{\operatorname{sat}(\hat{\mathbf{R}})} \\
\Delta \tau_{i j}^{e} & =\frac{1}{\operatorname{eff}(\hat{\mathbf{R}})}
\end{aligned}
$$

where $\operatorname{sat}(\hat{\mathbf{R}})$ and $\operatorname{eff}(\hat{\mathbf{R}})$ are the evaluations of the best solution (see Eqs. (2) and (3)) with respect to each objective.

For example, Figure 3(a) shows a fully connected directed graph for a backlog with six requirements $\mathbf{R}=\left\{r_{1}, r_{2}, r_{3}, r_{4}, r_{5}, r_{6}\right\}$ with associated development efforts and customers satisfaction sets, $\mathbf{E}=\{3,4,2,1,4,1\}, \mathbf{S}=$ $\{1,2,3,2,5,4\}$, respectively. Also, consider the set of functional dependencies $\mathbf{F}=\left\{r_{1} \Rightarrow r_{3}, r_{1} \Rightarrow r_{5}, r_{2} \Rightarrow r_{4}, r_{2} \Rightarrow r_{5}, r_{5} \Rightarrow r_{6}, r_{3} \odot r_{4}, r_{4} \oplus r_{5}\right\}$ and that the development effort bound $B$ is set to a value of 11 . Figures $3(\mathrm{~b})$ to $3(\mathrm{~d})$ depict the steps followed by ant $k$ during an iteration. Initially (see Figure $3(\mathrm{~b})$ ), the ant chooses randomly a requirement from the set $\left\{r_{1}, r_{2}\right\}$ that contains the visible requirements (i.e. those verifying requirements interactions and whose effort limit is lower than $B$ ). Suppose that it selects $r_{2}$ as the initial node and adds it to its solution, $\mathbf{R}_{k}=\left\{r_{2}\right\}$. Then the ant obtains the set of non visited neighbors nodes from $r_{2}$ is $N_{2}^{k}=\left\{r_{1}\right\}$ and arcs reaching to $r_{2}$ have 
been deleted because they could never be used. In this example, we are going to assume that the ant only uses heuristic information in order to build its solution $\mathbf{R}_{k}$, so at each step it will choose the vertex with the highest $\mu_{i j}$ value from the set of non visited neighbors nodes. Now the ant adds $r_{1}$ to its solution $\mathbf{R}_{k}=\left\{r_{2}, r_{1}\right\}$ and searches for a new requirement to add from this node as it is depicted in Figure 3(c). In this situation the ant's set of neighbors nodes is $N_{1}^{k}=\left\{r_{3}-r_{4}, r_{5}\right\}$ and using only heuristic information the next node to travel to is $r_{3}-r_{4}$ (note that this node is consequence of the combination interaction $r_{3} \odot r_{4}$. Finally, Figure 3(d) shows that once the ant has added $r_{3}-r_{4}$ to its solution, $\mathbf{R}_{k}=\left\{r_{2}, r_{1}, r_{3}, r_{4}\right\}$, it has to stop because there are not any other visible vertices due to the exclusion relationship $r_{4} \oplus r_{5}$.

\section{Experimental Evaluation}

In this section, we describe the aspects related with the design of the experiments for making the performance evaluation of the multi-objective ACO algorithm proposed. First, we present the data used in the experiments. Then, we briefly describe other two metaheuristic algorithms used in the experiments. Finally, we define the set of quality measures applied and the comparison methodology we have followed.

\subsection{Datasets}

For testing the effectiveness of our proposal ACS we have used two datasets. The first dataset is taken from Greer and Ruhe (2004). It has 20 requirements and 5 customers. The development effort associated to each requirement and the level of priority or value assigned by each customer to each requirement are shown in Table 1. The customers' weights are given in the 1 to 5 range, following a uniform distribution (see first row in Table 2). These values (and also those of the level of priority of each requirement) can be understood as linguistic labels such as: without importance (1), less important (2), important (3), very important (4), extremely important (5). Each requirements has an associate effort estimate in term of score between 1 and 10. Also, we consider that all requirements are independent. It is the same type of representation that has been previously used by Leguizamon and Michalewicz (1999); Fidanova (2005); Shi (2006) to tackle the 0/1 knapsack problem. This special case, denoted by Bagnall et al (2001) as basic NRP, allows us to reformulate any other NRP problem by simply preprocessing requirements, it is shown in Section 2. The main reason to use this dataset resides in its wide use in the evaluation of other studies of distinct instances of NRP (Finkelstein et al, 2009; Zhang et al, 2007; Durillo et al, 2009) and, as far as we know, the lack of other available real datasets due to the privacy policies followed by software development companies. At the time of performing the experiments we have set the development effort boundary as a percentage of the total development 
Table 1 Dataset 1, assignment of the priority level of each requirement, requirements development effort and interactions

\begin{tabular}{|c|c|c|c|c|c|c|c|c|c|c|c|c|c|c|c|c|c|c|c|c|}
\hline & $r_{1}$ & $r_{2}$ & $r_{3}$ & $r_{4}$ & $r_{5}$ & $r_{6}$ & $r_{7}$ & $r_{8}$ & $r_{9}$ & $r_{10}$ & $r_{11}$ & $r_{12}$ & $r_{13}$ & $r_{14}$ & $r_{15}$ & $r_{16}$ & $r_{17}$ & $r_{18}$ & $r_{19}$ & $r_{20}$ \\
\hline$c_{1}$ & 4 & 2 & 1 & 2 & 5 & 5 & 2 & 4 & 4 & 4 & 2 & 3 & 4 & 2 & 4 & 4 & 4 & 1 & 3 & 2 \\
\hline$c_{2}$ & 4 & 4 & 2 & 2 & 4 & 5 & 1 & 4 & 4 & 5 & 2 & 3 & 2 & 4 & 4 & 2 & 3 & 2 & 3 & 1 \\
\hline$c_{3}$ & 5 & 3 & 3 & 3 & 4 & 5 & 2 & 4 & 4 & 4 & 2 & 4 & 1 & 5 & 4 & 1 & 2 & 3 & 3 & 2 \\
\hline$c_{4}$ & 4 & 5 & 2 & 3 & 3 & 4 & 2 & 4 & 2 & 3 & 5 & 2 & 3 & 2 & 4 & 3 & 5 & 4 & 3 & 2 \\
\hline$c_{5}$ & 5 & 4 & 2 & 4 & 5 & 4 & 2 & 4 & 5 & 2 & 4 & 5 & 3 & 4 & 4 & 1 & 1 & 2 & 4 & 1 \\
\hline Eff. & 1 & 4 & 2 & 3 & 4 & 7 & 10 & 2 & 1 & 3 & 2 & 5 & 8 & 2 & 1 & 4 & 10 & 4 & 8 & 4 \\
\hline \multicolumn{21}{|c|}{$r_{3} \odot r_{12} r_{11} \odot r_{13}$} \\
\hline
\end{tabular}

Table 2 Customers relative importance

\begin{tabular}{lccccc}
\hline Customers' weights & $c_{1}$ & $c_{2}$ & $c_{3}$ & $c_{4}$ & $c_{5}$ \\
\hline For Dataset 1 & 1 & 4 & 2 & 3 & 4 \\
For Dataset 2 & 1 & 5 & 3 & 3 & 1 \\
\hline
\end{tabular}

effort needed to include all the requirements in a software product. Then we have considered the $30 \%, 50 \%$ and $70 \%$ of the total development effort, which respectively translates into an effort bound of 25, 43 and 60 effort units in our experiments.

The second dataset has been generated randomly with 100 requirement and 5 customers, according to the NRP model (see Table 3 ). The relative importance of the customers is given in the second row of Table 2. This dataset was defined because in real agile software projects development, in the initial timeboxes, we are faced with the problem of selecting requirements from a wider set. Therefore, the number of requirements has been incremented from 20 to 100 . The development effort values of each requirement are given in the 1 to 20 range. We had fixed 20 units (4 weeks) as the maximum development effort for a requirement, considering the timebox limit defined in agile methods (e.g. Scrum proposes iteration in the range 2 a 4 weeks). Related to the value, when customers have to make an assignment of the benefit that will imply the inclusion of a given requirement, they prefer to use a coarse grained scale instead of one of finer granularity. Usually they simply place requirements in one of three categories: inessential, desirable or mandatory (Wiegers, 2003; Simmons, 2004). Due to this last fact, the customers values of level of priority of requirements are in the range of 1 to 3 . Following the same considerations made on dataset 1 , we have set the development effort boundary using the same percentages $(30 \%, 50 \%$ and $70 \%)$ of the total development effort needed to include all the requirements in a software product, which respectively translates into an effort bound of 312, 519 and 726 effort units in our experiments. So, we will test ACS using six basic instances of NRP. 
Table 3 Dataset 2, assignment of the priority level of each requirement, requirements development effort and interactions

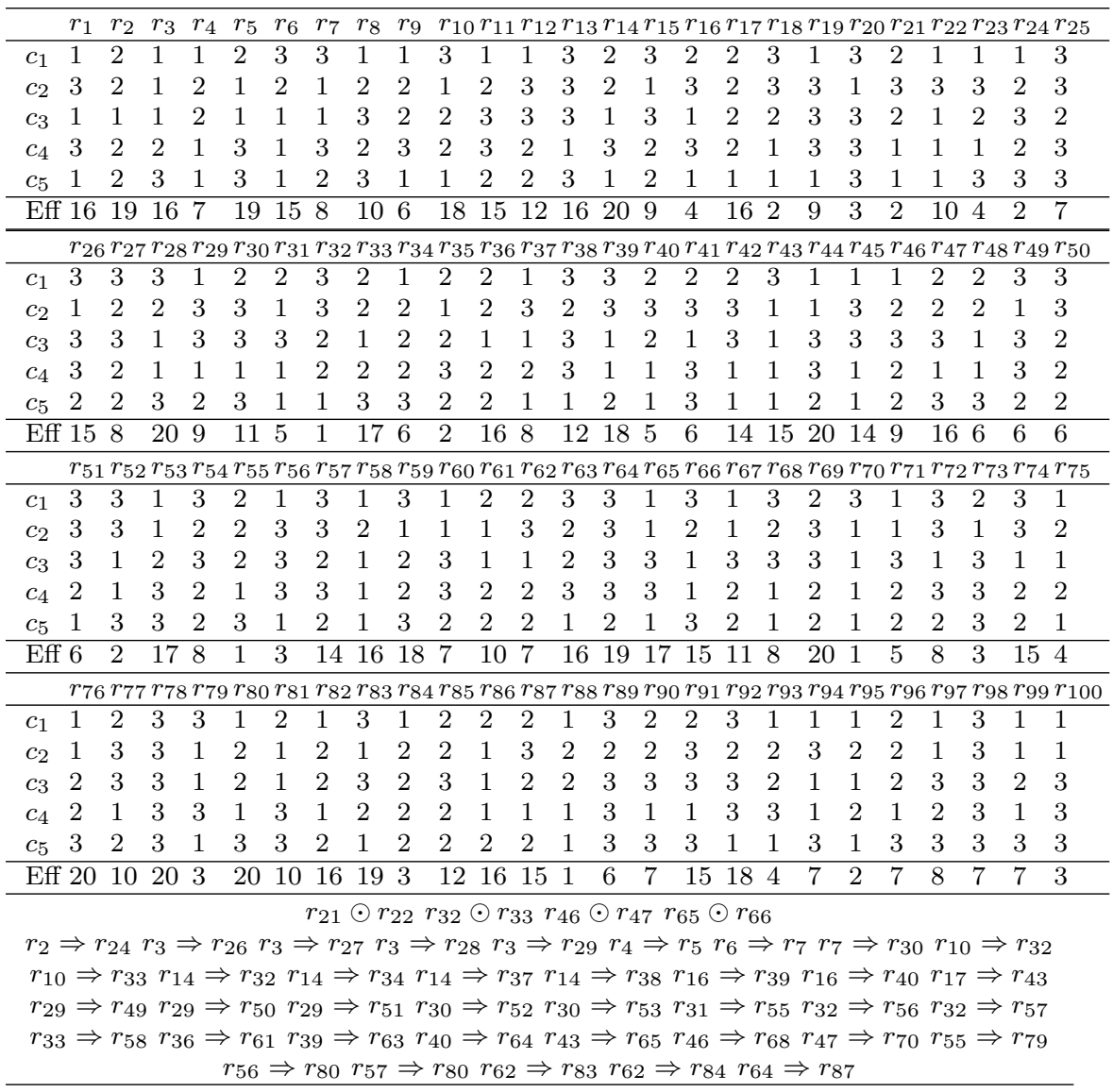

4.2 Metaheuristic techniques applied in experimentation

Many metaheuristic techniques have been applied to the requirement selection problem, a review of them can be found at (del Sagrado et al, 2010b). We select two algorithms against with our multi-objective ant colony system will be evaluated for solving the NRP: Greedy Randomized Adaptive Search Procedure (GRASP) and Non-dominated Sorting Genetic Algorithm (NSGA-II).

The first one is a metaheuristic algorithm that generates a good approximation to the efficient set of solutions of a multi-objective combinatorial optimization problem. GRASP was first introduced by Feo and Resende (1989). Survey papers on GRASP include Feo and Resende (1995), Pitsoulis and Resende (2003), and Resende and Ribeiro (2003).

GRASP proceeds iteratively by first building a greedy randomized solution and then improving it through a local search. The greedy randomized solution 
is built from a list of elements ranked by a greedy function by adding elements to the problem's solution set. The greedy function is in charge of measuring the profit of including an element in the solution with respect to the cost of its inclusion. In our approach, the greedy function used measures the quality of a requirement based on users satisfaction with respect to the effort, (i.e. $s_{i} / e_{i}$ ). In the our case the greedy function used measures the quality of a requirement $r_{i}$ based on users satisfaction with respect to the effort is the equation:

$$
\frac{\lambda_{o=s} * s_{i}+\frac{\lambda_{o=e}}{e_{i}}}{2 * e_{i}}
$$

where $\lambda_{o}$ are the weights associated with each objective as defined. This measure is a productivity metric that weighs the profit of including a requirement with respect to the resources involved in their development. The local search acts, in an iterative way, trying to replace the current solution by a better one located in its neighborhood. GRASP terminates when no better solution can be found in the neighborhood.

The fast Elitist Non-dominated Sorting Genetic algorithm (NSGA-II) was proposed by Deb et al (2002). The word elitist refers to the fact that only the best individuals found so far are transferred to the next population. During each generation, a population is constructed by combining the parent and the child population. A fitness value is assigned to all non-dominated solutions of the combined population according to its level of non-dominance. Thus, the dominated solutions have a fitness value less than that of any non dominated solution. During this process the fitness value should decrease to lead the search towards non dominated solutions. Individuals represent the possible solutions, that is, an individual is a set of requirements. Two fitness functions have been implemented (one per objective), considering that the NSGA-II algorithm will try to minimize each one. Each generation represents the evolution of the population. The idea is that by means of crossover and mutation the new children and mutated individuals have even better fitness values than the original ones. Better individuals have a higher probability of stating at the Pareto front, whose cardinality is limited by the population defined in the execution. In the case of NRP, the crossover and mutation methods are more specific and difficult than in other problems because we need to take into account the resources bound in order to obtain new valid individuals (see del Sagrado et al (2010a)). The works of Durillo et al (2011) and Zhang and Harman (2010) show that NSGA-II can solve NRP offering a set of comparable solutions with those obtained by other metaheuristics.

\subsection{Performance measures}

We have calculated the optimal Pareto front of the problem defined by the first dataset, in order to compare it against those calculated by the metaheuristic optimization techniques. However, when the number of requirements grows, dataset 2, the obtaining of the optimal Pareto front is an intractable problem. 
So, our approach is to compare the results using several quality indicators, giving an insight on the quality of the results achieved for all the executed algorithms. Therefore, we use the following indicators with the purpose of conducting a comparative measure of diversity and convergence of the solutions obtained:

- The number of non dominated solutions found (\#Solutions). Pareto fronts with a higher number of non dominated solutions are preferred.

- The size of the space covered by the set on non-dominated solutions found (Hypervolume) (Zitzler and Thiele, 1999). For a two dimensional problem, for each solution $i \in Q$, a vector $v_{i}$ is built with respect to a reference point $W$, and the solution $i$ is considered as the diagonal corner of an hypercube. Hypervolume is the volume occupied by the union of all of these hypercubes:

$$
H V=\operatorname{volume}\left(\bigcup_{i=1}^{|Q|} v_{i}\right)
$$

Pareto fronts whit higher hypervolume values are preferred.

- The extent of spread achieved among the obtained solutions ( $\Delta$-Spread) (Durillo et al, 2009).

$$
\Delta(F)=\frac{d_{f}+d_{l}+\sum_{i=1}^{n}\left|d_{i}-\bar{d}\right|}{d_{f}+d_{l}+(n-1) * \bar{d}}
$$

where $d_{i}$ is the Euclidean distance between two consecutive solutions, $\bar{d}$ is the average of these distances, $d_{f}, d_{l}$ are the Euclidean distances to the extreme solutions of the optimal Pareto front, and $n$ is the number of solutions in the obtained Pareto front $F$. Pareto fronts with smaller spread are preferred.

- A measure that evaluates the uniformity of the distribution of non-dominated solutions found (Spacing) (Schott, 1995). If the problem has $N$ objectives and its Pareto front has $n$ solutions, the spacing of $F$ is defined as:

$$
\operatorname{Spacing}(F)=\frac{\sum_{j=1}^{n}\left(\sum_{i=1}^{N}\left(1-\frac{\left|d_{i j}\right|}{\bar{d}_{i}}\right)^{2}\right)^{1 / 2}}{n * N}
$$

where, $\bar{d}_{i}$ is the mean value of the magnitude of the $i-t h$ objective in the set $F$. and $d_{i j}$ is the value of the $i$-th objective for the $j$-th solution in $F$. Pareto fronts with higher spacing are preferred.

Pareto fronts with a higher number of non-dominated solutions, smaller spread, higher spacing and higher hypervolume are preferred. The average and standard deviation of all of these quality indicators are computed. 


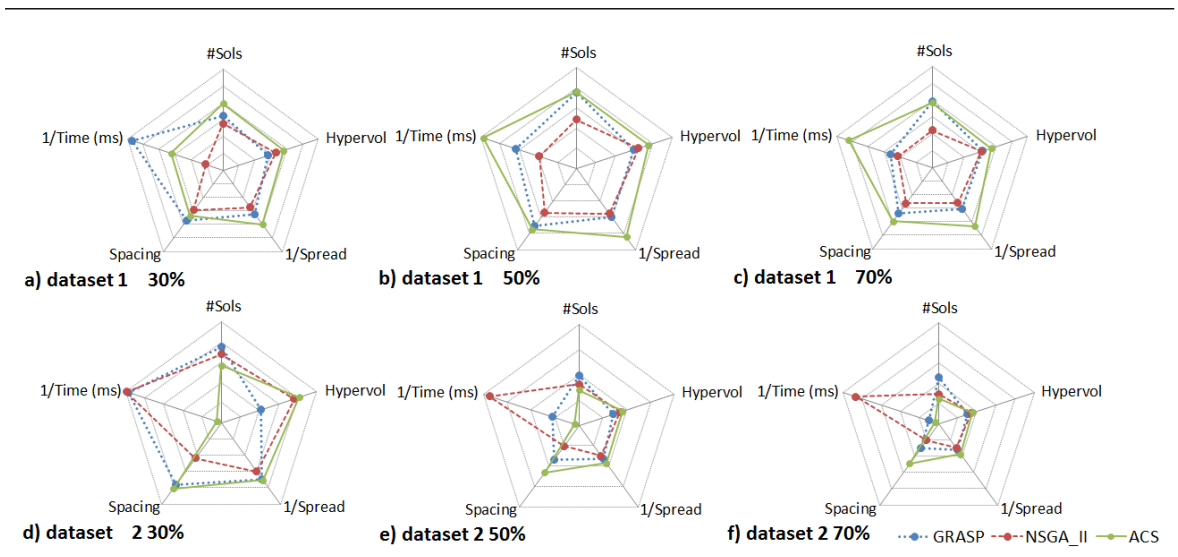

Fig. 4 Kiviat graph comparing metaheuristic algorithms

\subsection{Comparison Methodology}

The comparison of the results is done in four steps:

1. For each dataset and each development effort bound, we have performed one hundred consecutive executions of each of the metaheuristic search techniques, using different parameters settings. We compute the average and standard deviation of the number of solutions in the Pareto-front, of the quality indicators, and of the execution time.

2. With these values at hand, we have analyzed each technique separately. We make a numerical comparison of the different configurations of parameters used in order to show their influence on the behavior of the algorithms, selecting the parameters configuration that exhibits the best scores of the five quality indicators.

3. Once the best parameter setting has been established for each one of the algorithms (GRASP, NSGA-II and ACS), the average values of their indicators are visually compared, using Kiviat graphs (see Figure 4). This step allows us evaluate the goodness of our ACS proposal, comparing it against the other two search methods used.

4. The Pareto fronts returned by each metaheuristic search techniques are compared using the non-parametric significance Mann-Whitney U test, which is a non-parametric statistical hypothesis test for assessing whether one of two samples of independent observations tends to have larger values than the other. We compare the satisfaction dimension.

\section{Analysis of the Experimental Results}

Tables 5, 6 and 7, included in the appendix, 


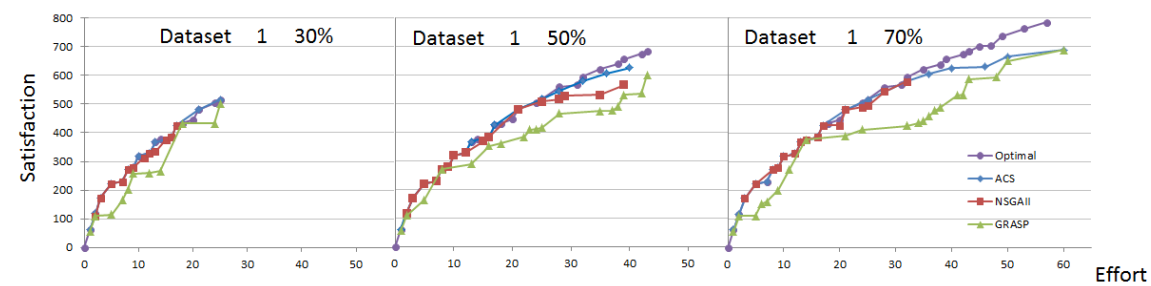

Fig. 5 Pareto fronts for dataset 1

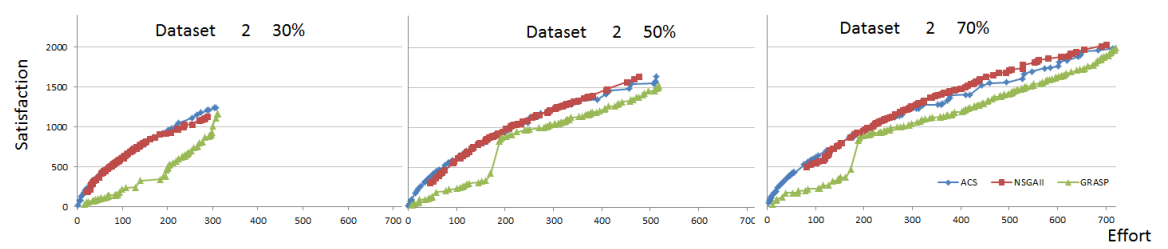

Fig. 6 Pareto fronts for dataset 2

\section{Appendix}

Tables of this appendix collect the data related to the execution of the algoritms.

Acknowledgements This research has been funded by the Spanish Ministry of Education, Culture and Sport under project TIN2010-20900-C04-02.

\section{References}

Bagnall AJ, Rayward-Smith VJ, Whittley I (2001) The next release problem. Information \& Software Technology 43(14):883-890

Baker P, Harman M, Steinhöfel K, Skaliotis A (2006) Search based approaches to component selection and prioritization for the next release problem. In: Proceedings of 22nd IEEE International Conference on Software Maintenance (ICSM 2006), Philadelphia, Pennsylvania, USA, IEEE Computer Society, pp 176-185

Boehm BW, Grübacher P, Briggs RO (2001) Easywinwin: A groupwaresupported nethodology for requirements negotiation. In: Proceedings of the 23rd International Conference on Software Engineering (ICSE 2001), Toronto, Ontario, Canada, IEEE Computer Society, pp 720-721

Carlshamre P (2002) Release planning in market-driven software product development: Provoking an understanding. Requirements Engineering $7(3): 139-151$

Carlshamre P, Sandahl K, Lindvall M, Regnell B, och Dag JN (2001) An industrial survey of requirements interdependencies in software product release planning. In: Proceedings of 5th IEEE International Symposium on 
Requirements Engineering (RE 2001), Toronto, Canada, IEEE Computer Society, pp 84-93

Cheng BHC, Atlee JM (2007) Research directions in requirements engineering. In: Proceedings of International Conference on Software Engineering, ISCE 2007, Workshop on the Future of Software Engineering (FOSE 2007), Minneapolis, MN, USA, pp 285-303

Coello CAC, Lamont GB, Veldhuizen DAV (2007) Evolutionary Algorithms for Solving Multi-Objective Problems Evolutionary Algorithms for Solving Multi-Objective Problems. Springer, New York

Davis AM (2003) The art of requirements triage. IEEE Computer 36(3):42-49

Deb K (2001) Multi-Objective Optimization Using Evolutionary Algorithms. John Wiley and Sons, New York

Deb K, Agrawal S, Pratap A, Meyarivan T (2002) A fast and elitist multiobjective genetic algorithm: NSGA-II. IEEE Trans Evolutionary Computation 6(2):182-197

Doerner KF, Gutjahr WJ, Hartl RF, Strauss C, Stummer C (2004) Pareto ant colony optimization: A metaheuristic approach to multiobjective portfolio selection. Annals Operational Research 131(1-4):79-99

Dorigo M, Gambardella LM (1997) Ant colony system: a cooperative learning approach to the traveling salesman problem. IEEE Trans Evolutionary Computation 1(1):53-66

Dorigo M, Stützle T (2004) Ant Colony Optimization. MIT Press, Cambridge, MA

Dorigo M, Birattari M, Stutzle T (2006) Ant colony optimization. IEEE Computational Intelligence Magazine 1(4):28-39

Durillo J, Zhang Y, Alba E, Nebro A (2009) A study of the multi-objective next release problem. In: Proceeding of 1st International Symposium on Search Based Software Engineering (SSBSE 2009), Cumberland Lodge, Windsor, UK, pp 49-58

Durillo JJ, Zhang Y, Alba E, Harman M, Nebro AJ (2011) A study of the biobjective next release problem. Empirical Software Engineering 16(1):29-60

Feo T, Resende M (1995) Greedy randomized adaptive search procedures. Journal of Global Optimization 6:109-133

Feo TA, Resende MGC (1989) A probabilistic heuristic for a computationally difficult set covering problem. Operations Research Letters 8(2):67-71

Fidanova S (2005) Ant colony optimization for multiple knapsack problem and model bias. In: Proceedings of the Third international conference on Numerical Analysis and its Applications (NAA'04), Rousse, Bulgaria, SpringerVerlag, pp 280-287

Finkelstein A, Harman M, Mansouri SA, Ren J, Zhang Y (2008) "fairness analysis" in requirements assignments. In: Proceeding of 16th IEEE International Requirements Engineering Conference (RE 2008), Barcelona, Spain, pp 115-124

Finkelstein A, Harman M, Mansouri SA, Ren J, Zhang Y (2009) A search based approach to fairness analysis in requirement assignments to aid negotiation, mediation and decision making. Requirement Engineering 14(4):231-245 
Greer D, Ruhe G (2004) Software release planning: an evolutionary and iterative approach. Information \& Software Technology 46(4):243-253

Häckel S, Fischer M, Zechel D, Teich T (2008) A multi-objective ant colony approach for pareto-optimization using dynamic programming. In: Proceedings of Genetic and Evolutionary Computation Conference (GECCO 2008), Atlanta, GA, USA, ACM, pp 33-40

Harman M, Jones BF (2001) Search-based software engineering. Information and Software Technology 43(14):833-839

Iredi S, Merkle D, Middendorf M (2001) Bi-criterion optimization with multi colony ant algorithms. In: Proceedings of Evolutionary Multi-Criterion Optimization, First International Conference (EMO 2001), Zurich, Switzerland, pp 359-372

Jiang H, Zhang J, Xuan J, Re Z, Hu Y (2010) A hybrid aco algorithm for the next release problem. In: Proceedings of the 2nd International Conference on Software Engineering and Data Mining (SEDM '10), IEEE, Chengdu, China, pp 166-171

Johnson J (2003) CHAOS chronicles v3.0. Tech. rep.

Karlsson J, Olsson S, Ryan K (1997) Improving practical support for largescale requirement prioritising. Requirement Engineering 2(1):51-60

Kotonya G, Sommerville I (1998) Requirements Engineering: Processes and Techniques. Wiley

Leguizamon G, Michalewicz Z (1999) A new version of ant system for subset problems. In: Proceedings of the 1999 Congress on Evolutionary Computation (CEC 99), Washington, DC USA, vol 2, pp 3 vol. (xxxvii+2348)

Momoh J, Ruhe G (2006) Release planning process improvement an industrial case study. Software Process: Improvement and Practice 11(3):295-307

Pitsoulis L, Resende M (2003) Greedy randomized adaptive search procedures, Oxford University Press, pp 168-183

Resende M, Ribeiro C (2003) Greedy randomized adaptive search procedures, Kluwer Academic Publishers, Dordrecht, pp 219-249

Ruhe G, Saliu MO (2005) The art and science of software release planning. IEEE Software 22(6):47-53

del Sagrado J, del Águila IM (2009) Ant colony optimization for requirement selection in incremental software development. In: Proceedings of the 1st International Symposium on Search Based Software Engineering (SSBSE '09), IEEE, Cumberland Lodge, Windsor, UK

del Sagrado J, del Águila I, Orellana F (2010a) Ant colony optimization for the next release problem: A comparative study. In: Proceeding of Second International Symposium on Search Based Software Engineering (SSBSE 2010), Benevento, Italy, pp $67-76$

del Sagrado J, del Águila IM, Orellana FJ, Túnez S (2010b) Requirements selection: Knowledge based optimization techniques for solving the next release problem. In: Proceedings of the 6th Workshop on Knowledge Engineering and Software Engineering (KESE6), Karlsruhe, Germany, CEURWS.org, CEUR Workshop Proceedings, vol 636 
del Sagrado J, del Águila IM, Orellana FJ (2011) Requirements interaction in the next release problem. In: Proceedings of 13th Annual Genetic and Evolutionary Computation Conference (GECCO 2011), Dublin, Ireland, pp 241-242

del Sagrado J, del Águila IM, Orellana FJ (2012) Metaheuristic aided software features assembly. In: Prodeeding of 20th European Conference on Artificial Intelligence (ECAI 2012) Including Prestigious Applications of Artificial Intelligence (PAIS-2012) System Demonstrations Track, Montpellier, France, pp 1009-1010

Saliu MO, Ruhe G (2007) Bi-objective release planning for evolving software systems. In: Proceedings of the 6th joint meeting of the European Software Engineering Conference and the ACM SIGSOFT International Symposium on Foundations of Software Engineering, Dubrovnik, Croatia, pp 105-114

Schott J (1995) Fault tolerant design using single and multicriteria genetic algorithm optimization. PhD thesis, Massachusetts Institute of Technology, M.S., USA

Shi H (2006) Solution to 0/1 knapsack problem based on improved ant colony algorithm. In: Proceeding of IEEE International Conference on Information Acquisition, Shandong, China, pp $1062-1066$

Simmons E (2004) Requirements triage: What can we learn from a "medical" approach? IEEE Software 21(4):86-88

de Souza JT, Maia CLB, Ferreira T, do Carmo RAF, Brasil M (2011) An ant colony optimization approach to the software release planning with dependent requirements. In: Proceedings of the 3rd International Symposium on Search Based Software Engineering (SSBSE '11), Springer, Szeged, Hungary, vol 6956, pp 142-157

Srinivas N, Deb K (1994) Muiltiobjective optimization using nondominated sorting in genetic algorithms. Evoltionary Computation 2:221-248

Wiegers KE (2003) Software Requirements. Microsoft Press, Redmon, WA, USA

Zhang Y, Harman M (2010) Search based optimization of requirements interaction management. In: Proceeding of Second International Symposium on Search Based Software Engineering (SSBSE 2010), Benevento, Italy, pp 47 $-56$

Zhang Y, Harman M, Mansouri SA (2007) The multi-objective next release problem. In: Proceedings of Genetic and Evolutionary Computation Conference (GECCO 2007), London, England, UK, pp 1129-1137

Zitzler E, Thiele L (1999) Multiobjective evolutionary algorithms: a comparative case study and the strength pareto approach. IEEE Trans Evolutionary Computation 3(4):257-271 
Table 4 Best result for each algorithm and dataset

\begin{tabular}{|c|c|c|c|c|c|}
\hline & \# Sols & Hypervol & $\Delta$ Spread & Spacing & Exec. Time (ms) \\
\hline \multicolumn{6}{|c|}{ Dataset $130 \%$} \\
\hline GRASP & $11.37 \pm 1.47$ & $5,851.00 \pm 277.82$ & $0.64 \pm 0.09$ & $0.36 \pm 0.03$ & $362.80 \pm 10.84$ \\
\hline NSGA-II & $9.69 \pm 2.09$ & $6,842.92 \pm 849.03$ & $0.76 \pm 0.09$ & $0.29 \pm 0.11$ & $1,891.55 \pm 196.10$ \\
\hline $\mathrm{ACS}$ & $13.80 \pm 13.80$ & $7,817.44 \pm 38.32$ & $0.52 \pm 0.02$ & $0.33 \pm 0.01$ & $637.69 \pm 21.42$ \\
\hline \multicolumn{6}{|c|}{ Dataset $150 \%$} \\
\hline GRASP & $17.65 \pm 2.22$ & $14,508.20 \pm 265.88$ & $0.73 \pm 0.07$ & $0.35 \pm 0.03$ & $1,208.25 \pm 29.15$ \\
\hline NSGA-II & $11.30 \pm 1.82$ & $15,676.77 \pm 1,214.25$ & $0.79 \pm 0.07$ & $0.27 \pm 0.06$ & $1,980.93 \pm 144.42$ \\
\hline $\mathrm{ACS}$ & $\mathbf{1 7 . 7 5} \pm 0.61$ & $18,153.33 \pm 51.26$ & $0.52 \pm 0.01$ & $0.37 \pm 0.01$ & $787.62 \pm 33.29$ \\
\hline \multicolumn{6}{|c|}{ Dataset $170 \%$} \\
\hline GRASP & $20.97 \pm 2.16$ & $24,837.24 \pm 297.55$ & $0.68 \pm 0.06$ & $0.34 \pm 0.03$ & $1,678.75 \pm 41.20$ \\
\hline NSGA-II & $11.70 \pm 1.90$ & $24,408.67 \pm 1,746.18$ & $0.80 \pm 0.07$ & $0.26 \pm 0.05$ & $2,034.25 \pm 120.81$ \\
\hline $\mathrm{ACS}$ & $20.57 \pm 20.57$ & $29,196.12 \pm 53.72$ & $0.48 \pm 0.02$ & $0.40 \pm 0.01$ & $836.76 \pm 34.35$ \\
\hline \multicolumn{6}{|c|}{ Dataset $230 \%$} \\
\hline GRASP & $62.74 \pm 4.97$ & $119,395.37 \pm 826.79$ & $0.69 \pm 0.05$ & $0.39 \pm 0.02$ & $29,141.69 \pm 638.77$ \\
\hline NSGA-II & $56.86 \pm 8.91$ & $219,662.51 \pm 6,320.36$ & $0.81 \pm 0.07$ & $0.22 \pm 0.04$ & $28,428.73 \pm 1,385.88$ \\
\hline $\mathrm{ACS}$ & $47.41 \pm 5.87$ & $234,609.66 \pm 1,689.93$ & $0.69 \pm 0.06$ & $0.41 \pm 0.04$ & $618,755.93 \pm 26,695.67$ \\
\hline \multicolumn{6}{|c|}{ Dataset $250 \%$} \\
\hline GRASP & $81.39 \pm 5.85$ & $419,804.19 \pm 1,617.48$ & $0.74 \pm 0.04$ & $0.31 \pm 0.02$ & $120,585.52 \pm 4,053.31$ \\
\hline NSGA-II & $67.00 \pm 11.24$ & $497,866.51 \pm 16,045.67$ & $0.81 \pm 0.06$ & $0.19 \pm 0.03$ & $35,149.95 \pm 743.31$ \\
\hline $\mathrm{ACS}$ & $57.68 \pm 5.69$ & $527,685.22 \pm 2,738.50$ & $0.66 \pm 0.06$ & $0.43 \pm 0.04$ & $770,221.21 \pm 37,331.72$ \\
\hline \multicolumn{6}{|c|}{ Dataset $270 \%$} \\
\hline GRASP & $130.88 \pm 5.89$ & $762,924.13 \pm 1,822.50$ & $0.71 \pm 0.03$ & $0.28 \pm 0.02$ & $336,762.35 \pm 13,095.81$ \\
\hline NSGA-II & $83.32 \pm 10.52$ & $873,383.61 \pm 24,556.01$ & $0.77 \pm 0.05$ & $0.19 \pm 0.03$ & $38,295.95 \pm 784.52$ \\
\hline $\mathrm{ACS}$ & $70.98 \pm 5.27$ & $902,769.29 \pm 3,141.76$ & $0.61 \pm 0.06$ & $0.45 \pm 0.04$ & $881,950.91 \pm 54,188.14$ \\
\hline
\end{tabular}


Table 5 Results Greedy Randomized Adaptive Search Procedure (GRASP)

\begin{tabular}{|c|c|c|c|c|c|}
\hline $\begin{array}{l}\text { Parameters } \\
(\text { iter }, \alpha)\end{array}$ & \# Sols & Hypervol & $\Delta$ Spread & Spacing & $\begin{array}{l}\text { Exec. Time } \\
(\mathrm{ms})\end{array}$ \\
\hline \multicolumn{6}{|c|}{ Dataset $130 \%$} \\
\hline$(1000,0.9)$ & $11.37 \pm 1.47$ & $5,851.00 \pm 277.82$ & $0.64 \pm 0.09$ & $0.36 \pm 0.03$ & $362.80 \pm 10.84$ \\
\hline$(1000,0.8)$ & $11.67 \pm 1.60$ & $5,239.29 \pm 337.86$ & $0.71 \pm 0.09$ & $0.40 \pm 0.03$ & $356.41 \pm 11.28$ \\
\hline$(1000,0.5)$ & $6.72 \pm 1.03$ & $3,571.45 \pm 526.58$ & $0.75 \pm 0.10$ & $0.35 \pm 0.03$ & $340.61 \pm 10.12$ \\
\hline$(1000,0.3)$ & $6.00 \pm 0.00$ & $1,487.00 \pm 0.00$ & $0.65 \pm 0.00$ & $0.31 \pm 0.00$ & $339.06 \pm 11.66$ \\
\hline$(500,0.9)$ & $10.26 \pm 1.60$ & $5,386.75 \pm 318.31$ & $0.65 \pm 0.09$ & $0.38 \pm 0.03$ & $183.77 \pm 10.46$ \\
\hline$(500,0.8)$ & $11.12 \pm 1.48$ & $4,764.96 \pm 407.39$ & $0.72 \pm 0.09$ & $0.40 \pm 0.03$ & $180.64 \pm 11.42$ \\
\hline$(500,0.5)$ & $6.56 \pm 1.00$ & $3,125.67 \pm 471.71$ & $0.82 \pm 0.07$ & $0.35 \pm 0.04$ & $\mathbf{1 6 9 . 5 4} \pm \mathbf{9 . 2 8}$ \\
\hline$(500,0.3)$ & $6.00 \pm 0.00$ & $1,487.00 \pm 0.00$ & $0.65 \pm 0.00$ & $0.31 \pm 0.00$ & $169.99 \pm 9.75$ \\
\hline \multicolumn{6}{|c|}{ Dataset $150 \%$} \\
\hline$(1000,0.9)$ & $17.65 \pm 2.22$ & $14,508.20 \pm 265.88$ & $\mathbf{0 . 7 3} \pm \mathbf{0 . 0 7}$ & $0.35 \pm 0.03$ & $1,208.25 \pm 29.15$ \\
\hline$(1000,0.8)$ & $17.33 \pm 2.04$ & $13,730.69 \pm 365.03$ & $0.77 \pm 0.06$ & $0.40 \pm 0.03$ & $1,202.66 \pm 24.38$ \\
\hline$(1000,0.5)$ & $14.96 \pm 0.60$ & $11,268.44 \pm 175.26$ & $0.78 \pm 0.04$ & $0.27 \pm 0.02$ & $1,175.61 \pm 23.76$ \\
\hline$(1000,0.3)$ & $14.00 \pm 0.00$ & $9,847.00 \pm 0.00$ & $0.80 \pm 0.00$ & $0.23 \pm 0.00$ & $1,162.34 \pm 23.35$ \\
\hline$(500,0.9)$ & $16.99 \pm 2.10$ & $13,932.90 \pm 368.64$ & $0.74 \pm 0.07$ & $0.35 \pm 0.03$ & $605.58 \pm 22.47$ \\
\hline$(500,0.8)$ & $16.66 \pm 2.01$ & $13,275.39 \pm 477.28$ & $0.77 \pm 0.07$ & 0.03 & $599.04 \pm 19.03$ \\
\hline$(500,0.5)$ & $14.64 \pm 0.63$ & $11,122.17 \pm 184.16$ & $0.79 \pm 0.03$ & $0.26 \pm 0.02$ & $584.37 \pm 16.30$ \\
\hline$(500,0.3)$ & $14.00 \pm 0.00$ & $9,847.00 \pm 0.00$ & $0.80 \pm 0.00$ & $0.23 \pm 0.00$ & $580.63 \pm 18.71$ \\
\hline \multicolumn{6}{|c|}{ Dataset $170 \%$} \\
\hline$(1000,0.3)$ & $20.97 \pm 2.16$ & $24,837.24 \pm 297.55$ & $0.68 \pm 0.06$ & $0.34 \pm 0.03$ & $1,678.75 \pm 41.20$ \\
\hline$(1000,0.8)$ & $19.67 \pm 1.71$ & $23,897.72 \pm 309.97$ & $0.74 \pm 0.05$ & $0.38 \pm 0.03$ & $1,531.68 \pm 38.51$ \\
\hline$(1000,0.5)$ & $14.89 \pm 0.68$ & $21,232.90 \pm 161.44$ & $0.78 \pm 0.04$ & $0.27 \pm 0.02$ & $1,170.96 \pm 26.27$ \\
\hline$(1000,0.3)$ & $14.00 \pm 0.00$ & $19,826.00 \pm 0.00$ & $0.80 \pm 0.00$ & $0.23 \pm 0.00$ & $1,160.77 \pm 24.36$ \\
\hline$(500,0.9)$ & $20.26 \pm 2.18$ & $24,473.66 \pm 377.05$ & $0.69 \pm 0.06$ & $0.34 \pm 0.03$ & $839.84 \pm 31.48$ \\
\hline$(500,0.8)$ & $19.48 \pm 1.59$ & $3.63 \pm 387.34$ & $0.75=$ & 0.03 & $4 \pm 29.40$ \\
\hline$(500,0.5)$ & $14.70 \pm 0.63$ & $21,074.26 \pm 162.06$ & $0.80 \pm 0.04$ & $0.26 \pm 0.02$ & $588.96 \pm 18.68$ \\
\hline$(500,0.3)$ & $14.00 \pm 0.00$ & $19,826.00 \pm 0.00$ & $0.80 \pm 0.00$ & $0.23 \pm 0.00$ & $\mathbf{5 8 4 . 5 0} \pm \mathbf{1 7 . 6 4}$ \\
\hline \multicolumn{6}{|c|}{ Dataset $230 \%$} \\
\hline$(500,0.3)$ & $53.99 \pm 3.78$ & $111,783.21 \pm 296.19$ & $\mathbf{0 . 5 5} \pm \mathbf{0 . 0 5}$ & $0.40 \pm 0.02$ & $15,715.18 \pm 1,619.54$ \\
\hline$(500,0.5)$ & $56.21 \pm 4.79$ & $117,227.38 \pm 871.86$ & $0.65 \pm 0.05$ & $0.38 \pm 0.03$ & $15,291.71 \pm 1$, \\
\hline$(500,0.8)$ & $48.99 \pm 4.88$ & $126,148.96 \pm 1,824.04$ & $0.65 \pm 0.05$ & $0.34 \pm 0.04$ & $15,436.13 \pm 1,112.42$ \\
\hline$(500,0.9)$ & $46.32 \pm 3.87$ & $128,336.00 \pm 1,770.28$ & $0.64 \pm 0.05$ & $0.33 \pm 0.03$ & $14,674.33 \pm 235.43$ \\
\hline$(1000,0.3)$ & $57.99 \pm 3.66$ & $112,418.88 \pm 235.53$ & $0.60 \pm 0.04$ & 0.02 & $28,915.27 \pm 371.20$ \\
\hline$(1000,0.5)$ & $62.74 \pm 4.97$ & $119,395.37 \pm 826.79$ & $0.69 \pm 0.05$ & $0.39 \pm 0.02$ & $29,141.69 \pm 638.77$ \\
\hline$(1000,0.8)$ & $53.66 \pm 5.14$ & $129,680.62 \pm 1,501.14$ & $0.67 \pm 0.06$ & $0.35 \pm 0.03$ & $29,346.86 \pm 384.38$ \\
\hline$(1000,0.9)$ & $52.47 \pm 5.23$ & $132,182.64 \pm 1,924.53$ & $0.66 \pm 0.05$ & $0.34 \pm 0.03$ & $29,526.05 \pm 363.79$ \\
\hline \multicolumn{6}{|c|}{ Dataset $250 \%$} \\
\hline$(500,0.3)$ & $71.05 \pm 4.20$ & $402,416.59 \pm 1,039.90$ & $0.77 \pm 0.03$ & $0.41 \pm 0.02$ & $59,385.89 \pm 1,531.46$ \\
\hline$(500,0.5)$ & $69.32 \pm 5.01$ & $407,057.50 \pm 1,531.07$ & $0.74 \pm 0.04$ & $0.40 \pm 0.03$ & $61,738.85 \pm 4,761.45$ \\
\hline$(500,0.8)$ & $73.32 \pm 5.54$ & $415,226.09 \pm 1,785.76$ & $0.74 \pm 0.04$ & $0.31 \pm 0.03$ & $63,504.13 \pm 3,792.53$ \\
\hline$(500,0.9)$ & $67.99 \pm 5.32$ & $420,263.92 \pm 2,183.74$ & $0.74 \pm 0.04$ & $0.32 \pm 0.03$ & $61,463.12 \pm 1,935.45$ \\
\hline$(1000,0.3)$ & $75.80 \pm 4.69$ & $404,784.23 \pm 790.84$ & $0.79 \pm 0.03$ & $0.44 \pm 0.02$ & $121,349.99 \pm 2,508.53$ \\
\hline$(1000,0.5)$ & $74.82 \pm 4.50$ & $410,221.09 \pm 1,464.63$ & $\mathbf{0 . 7 4} \pm \mathbf{0 . 0 3}$ & $0.41 \pm 0.02$ & $122,381.05 \pm 3,872.40$ \\
\hline$(1000,0.8)$ & $\mathbf{8 1 . 3 9} \pm \mathbf{5 . 8 5}$ & $419,804.19 \pm 1,617.48$ & $0.74 \pm 0.04$ & $0.31 \pm 0.02$ & $120,585.52 \pm 4,053.31$ \\
\hline$(1000,0.9)$ & $75.81 \pm 5.81$ & $425,642.47 \pm 1,894.97$ & $0.74 \pm 0.05$ & $0.32 \pm 0.03$ & $118,335.71 \pm 2,000.27$ \\
\hline \multicolumn{6}{|c|}{ Dataset $270 \%$} \\
\hline$(500,0.3)$ & $120.51 \pm 5.59$ & $745,387.59 \pm 781.13$ & $0.74 \pm 0.03$ & $0.34 \pm 0.02$ & $164,035.28 \pm 8,251.6$ \\
\hline$(500,0.5)$ & $117.43 \pm 5.67$ & $750,964.34 \pm 1,601.37$ & $0.71 \pm 0.03$ & $0.32 \pm 0.02$ & $165,807.50 \pm 7,222.89$ \\
\hline$(500,0.8)$ & $118.35 \pm 7.19$ & $758,626.65 \pm 2,045.66$ & $0.71 \pm 0.03$ & $0.28 \pm 0.02$ & $173,550.47 \pm 14,891.71$ \\
\hline$(500,0.9)$ & $109.35 \pm 6.57$ & $763,586.43 \pm 2,015.47$ & $\mathbf{0 . 7 0} \pm \mathbf{0 . 0 3}$ & $0.28 \pm 0.02$ & $168,849.21 \pm 9,234.50$ \\
\hline$(1000,0.3)$ & $129.59 \pm 5.76$ & $747,684.46 \pm 773.36$ & $0.75 \pm 0.02$ & $0.34 \pm 0.02$ & $329,101.66 \pm 11,926.18$ \\
\hline$(1000,0.5)$ & $128.50 \pm 6.49$ & $754,667.97 \pm 1,647.21$ & $0.72 \pm 0.02$ & $0.32 \pm 0.02$ & $337,569.40 \pm 21,378.16$ \\
\hline$(1000,0.8)$ & $130.88 \pm 5.90$ & $762,924.13 \pm 1,822.50$ & $0.71 \pm 0.03$ & $0.28 \pm 0.02$ & $336,762.35 \pm 13,095.81$ \\
\hline$(1000,0.9)$ & $120.14 \pm 7.27$ & $769,613.34 \pm 2,064.60$ & $\mathbf{0 . 7 0} \pm 0.03$ & $0.29 \pm 0.02$ & $324,604.49 \pm 13,244.30$ \\
\hline
\end{tabular}


Table 6 Non-dominated Sorting Genetic Algorithm (NSGA-II)

\begin{tabular}{|c|c|c|c|c|c|}
\hline $\begin{array}{l}\text { Parameters } \\
\text { (Pop, } p_{\text {mut }}, \\
\left.p_{\text {cross }}, \text { Gen }\right)\end{array}$ & \# Sols & Hypervol & $\Delta$ Spread & Spacing & $\begin{array}{l}\text { Exec. Time } \\
(\mathrm{ms})\end{array}$ \\
\hline \multicolumn{6}{|c|}{ Dataset $130 \%$} \\
\hline$(20, .05, .9,500)$ & $7.83 \pm 1.70$ & $6,429.18 \pm 1,078.58$ & $0.76 \pm 0.11$ & $0.27 \pm 0.12$ & $1,105.33 \pm 124.41$ \\
\hline$(20, .05, .9,500)$ & $8.32 \pm 1.80$ & $6,623.22 \pm 802.87$ & $0.76 \pm 0.10$ & $0.27 \pm 0.11$ & $1,100.97 \pm 122.37$ \\
\hline$(40, .05, .9,250)$ & $9.69 \pm 2.09$ & $6,842.92 \pm 849.03$ & $0.76 \pm 0.09$ & $0.29 \pm 0.11$ & $1,891.55 \pm 196.10$ \\
\hline$(40, .05, .8,250)$ & $9.44 \pm 2.57$ & $6,663.56 \pm 935.59$ & $0.73 \pm 0.10$ & $0.33 \pm 0.13$ & $1,856.43 \pm 216.09$ \\
\hline \multicolumn{6}{|c|}{ Dataset $150 \%$} \\
\hline$(20, .05, .9,500)$ & $8.86 \pm 2.14$ & $14,832.53 \pm 1,957.58$ & $0.80 \pm 0.08$ & $0.26 \pm 0.10$ & $1,130.43 \pm 106.19$ \\
\hline$(20, .05, .8,500)$ & $9.38 \pm 2.15$ & $15,205.51 \pm 1,623.13$ & $0.79 \pm 0.07$ & $0.26 \pm 0.10$ & $1,137.50 \pm 99.64$ \\
\hline$(20, .05, .9,250)$ & $11.30 \pm 1.82$ & $15,676.77 \pm 1,214.25$ & $0.79 \pm 0.07$ & $0.27 \pm 0.07$ & $1,980.93 \pm 144.42$ \\
\hline$(20, .05, .8,250)$ & $11.46 \pm 1.98$ & $15,664.81 \pm 1,339.10$ & $0.80 \pm 0.06$ & $0.27 \pm 0.06$ & $1,999.39 \pm 138.59$ \\
\hline \multicolumn{6}{|c|}{ Dataset $170 \%$} \\
\hline$(20, .05, .9,500)$ & $9.11 \pm 1.61$ & $23,494.48 \pm 2,303.49$ & $0.80 \pm 0.09$ & $E 0.06$ & $1,144.04 \pm 95.96$ \\
\hline$(20, .05, .8,500)$ & $9.22 \pm 2.09$ & $23,358.35 \pm 3,061.03$ & $0.78 \pm 0.08$ & $0.26 \pm 0.10$ & $1,156.72 \pm 100.40$ \\
\hline$(40, .05, .9,250)$ & $11.33 \pm 1.93$ & $23,967.40 \pm 1,974.73$ & $0.81 \pm 0.06$ & $0.26 \pm 0.05$ & $2,035.61 \pm 123.05$ \\
\hline$(40, .05, .8,250)$ & $11.70 \pm 1.90$ & $24,408.67 \pm 1,746.18$ & $0.80 \pm 0.07$ & $0.26 \pm 0.05$ & $2,034.25 \pm 120.81$ \\
\hline \multicolumn{6}{|c|}{ Dataset $230 \%$} \\
\hline$(125, .01, .9,80)$ & $54.34 \pm 8.51$ & $218,138.21 \pm 6,861.82$ & 0.80 & $0.22 \pm 0.05$ & $28,127.77 \pm 1,275.83$ \\
\hline$(125, .01, .8,80)$ & $56.86 \pm 8.91$ & $219,662.51 \pm 6,320.36$ & $0.81 \pm 0.07$ & 0.04 & $28,428.73 \pm 1,385.88$ \\
\hline$(100, .01, .9,100)$ & $46.73 \pm 8.59$ & $213,287.75 \pm 9,791.80$ & $0.83 \pm 0.07$ & $0.20 \pm 0.06$ & $22,987.83 \pm 1,329.2$ \\
\hline$(100, .05, .8,100)$ & $50.79 \pm 8.12$ & $216,657.11 \pm 7,377.47$ & $0.82 \pm 0.06$ & $0.20 \pm 0.05$ & $24,134.12 \pm 1,546.03$ \\
\hline \multicolumn{6}{|c|}{ Dataset $250 \%$} \\
\hline$(125$ & $\mathbf{9} \pm \mathbf{8 . 5 4}$ & $542,408.15 \pm 22,993.2$ & $60.65 \pm 0.06$ & $0.14 \pm 0.02$ & $40,683.34 \pm 518.12$ \\
\hline$(125, .01, .9,80)$ & 11.86 & $495,948.91 \pm 14,310.53$ & $0.81 \pm 0.0$ & & $35,045.76 \pm 840.87$ \\
\hline$(125, .01,0.8,80$ & $67.00 \pm 11.2$ & $1497,866.51 \pm 16,045.6$ & & & $35,149.95 \pm 743.31$ \\
\hline$(100, .01, .9,100)$ & $53.26 \pm 10.04$ & $483,773.06 \pm 19,331.55$ & $0.85 \pm 0.07$ & $0.17 \pm 0.04$ & $28,453.78 \pm 708.03$ \\
\hline$(100, .05$ & $57.30 \pm 9.89$ & $488,446.02 \pm 19,717.84$ & $0.83 \pm 0.06$ & $0.17 \pm 0.04$ & $28,620.55 \pm 843.44$ \\
\hline \multicolumn{6}{|c|}{ Dataset 2 70\% } \\
\hline$(125, .01, .9,80)$ & $80.43 \pm 10.04$ & $868,208.57 \pm 24,183.01$ & \pm 0.05 & 0.03 & $38,716.48 \pm 1,681.94$ \\
\hline 01880 & $\pm \mathbf{1 0 . 5}$ & $3873,383.61 \pm 24,556.0$ & 10.77 & $0.19 \pm 0.03$ & $38,295.95 \pm 784.52$ \\
\hline$(100, .01, .9,100)$ & $68.06 \pm 6.99$ & $859,481.95 \pm 25,202.17$ & $0.80 \pm 0.06$ & $0.18 \pm 0.03$ & $31,283.26 \pm 581.24$ \\
\hline$(100, .05, .8,100)$ & $71.72 \pm 8.69$ & $870,345.56 \pm 23,093.39$ & $0.77 \pm 0.06$ & $0.19 \pm 0.03$ & $31,352.58 \pm 717.18$ \\
\hline
\end{tabular}


Table 7 Ant Colony System (ACS). Iteration $=100 \rho=0.01, q_{0}=0.95$

\begin{tabular}{|c|c|c|c|c|}
\hline $\begin{array}{l}\text { Parametssols } \\
(\text { Ants, } \alpha, \beta)\end{array}$ & Hypervol & $\Delta$ Spread & Spacing & $\begin{array}{l}\text { Exec. Time } \\
(\mathrm{ms})\end{array}$ \\
\hline \multicolumn{5}{|c|}{ Dataset $130 \%$} \\
\hline$\overline{(10,0,1) 13.80 \pm 0.51}$ & $7,817.44 \pm 38.32$ & $0.52 \pm 0.0$ & $\overline{20.33 \pm 0.01}$ & $637.69 \pm 21.42$ \\
\hline$(10,1,0) 12.56 \pm 1.00$ & $6,326.28 \pm 375.84$ & $0.52 \pm 0.08$ & $0.34 \pm 0.02$ & $533.57 \pm 23.97$ \\
\hline$(10,1,1) 13.66 \pm 0.59$ & $7,801.76 \pm 54.74$ & $0.52 \pm 0.03$ & $0.33 \pm 0.01$ & $641.09 \pm 21.85$ \\
\hline$(10,1,2) 13.66 \pm 0.61$ & $7,805.39 \pm 49.87$ & $0.52 \pm 0.03$ & $0.33 \pm 0.01$ & $639.10 \pm 17.79$ \\
\hline$(10,1,5) 13.69 \pm 0.61$ & $7,806.74 \pm 50.69$ & $0.52 \pm 0.03$ & $0.33 \pm 0.01$ & $744.67 \pm 23.54$ \\
\hline \multicolumn{5}{|c|}{ Dataset $150 \%$} \\
\hline$(10,0,1) 17.65 \pm 0.56$ & $18,144.53 \pm$ & $0.52 \pm 0.02$ & $0.37 \pm 0.01$ & $789.03 \pm 34.51$ \\
\hline$(10,1,0) 15.73 \pm 1.22$ & $14,951.09 \pm 603.70$ & $0.57 \pm 0.05$ & $0.37 \pm 0.02$ & $761.86 \pm 34.02$ \\
\hline$(10,1,1) 17.60 \pm 0.60$ & $18,143.78 \pm 56.05$ & $0.52 \pm 0.02$ & $0.37 \pm 0.01$ & $9 \pm 27.80$ \\
\hline$(10,1,2) 17.75 \pm 0.61$ & $18,153.33 \pm 51.26$ & $0.52 \pm 0.0$ & $\pm \mathbf{0 . 0 1}$ & $2 \pm 33.29$ \\
\hline$(10,1,5) 17.66 \pm 0.59$ & $18,140.09 \pm 64.45$ & $0.52 \pm 0.02$ & $\pm \mathbf{0 . 0 1}$ & $0 \pm 33.36$ \\
\hline \multicolumn{5}{|c|}{ Dataset $170 \%$} \\
\hline$(10,0,1) 20.57 \pm 0.64$ & $29,188.04 \pm 61.87$ & $0.49 \pm 0.02$ & \pm 0.01 & 84 \\
\hline$(10,1,0) 19.77 \pm 1.35$ & $.17 \pm 686.76$ & $0.53 \pm 0.05$ & \pm 0.02 & $3 \pm 3$ \\
\hline$(10,1,1) 20.57 \pm 0.62$ & $29,196.12 \pm 53.72$ & $0.48 \pm 0.0$ & \pm 0.01 & $6 \pm 3$ \\
\hline$(10,1,2) 20.53 \pm 0.74$ & $29,195.16 \pm 55.44$ & $0.48 \pm 0.0$ & $20.40 \pm 0.01$ & $2 \pm 31.57$ \\
\hline$(10,1,5) 20.50 \pm 0.73$ & $29,176.01 \pm 79.45$ & $0.49 \pm 0.02$ & $0.40 \pm 0$ & $969.39 \pm 44.37$ \\
\hline \multicolumn{5}{|c|}{ Dataset $230 \%$} \\
\hline $47.41 \pm 5.87$ & $234,609.66 \pm 1,689.93$ & $0.69 \pm 0.0$ & $=0.04$ & $5.93 \pm 2$ \\
\hline$(50,1,0) 38.12 \pm 5.15$ & $111,445.99 \pm 3,602.45$ & $0.79 \pm 0.06$ & $0.35 \pm 0.05$ & $487.56 \pm$ \\
\hline$(50,1,1) 45.74 \pm 5.65$ & $234,212.10 \pm 2,000.59$ & $0.69 \pm 0.0$ & $0.42 \pm 0.04$ & $630,320.35 \pm 31$ \\
\hline$(50,1,2) 47.12 \pm 5.44$ & $234,583.42 \pm 1,710.00$ & $0.68 \pm 0.06$ & $0.41 \pm 0.04$ & $616,873.55 \pm 21,983.20$ \\
\hline$(50,1,5) 45.30 \pm 4.72$ & $234,354.84 \pm 1,881.55$ & $0.68 \pm 0.07$ & $0.41 \pm 0.04$ & $646,635.17 \pm 30,6$ \\
\hline \multicolumn{5}{|c|}{ Dataset $250 \%$} \\
\hline$(50,0,1) 57.72 \pm 5.16$ & $527,407.75 \pm 3,107.23$ & $0.67 \pm 0.07$ & $0.44 \pm 0.04$ & $=771,106.11 \pm 37,794.04$ \\
\hline$(50,1,0) \mathbf{6 3 . 1 7} \pm \mathbf{5 . 8 9}$ & $334,719.66 \pm 5,059.43$ & $0.74 \pm 0.06$ & $0.34 \pm 0.03$ & $919,818.36 \pm 45,425.92$ \\
\hline$(50,1,1) 57.76 \pm 5.37$ & $527,577.63 \pm 3,002.42$ & $0.65 \pm 0.0$ & $70.43 \pm 0.03$ & $768,485.74 \pm 34,399.04$ \\
\hline$(\mathbf{5 0 , 1 , 2 ) 5 7 . 6 8 \pm 5 . 6 9}$ & $527,685.22 \pm 2,738.50$ & $0.66 \pm 0.06$ & $0.43 \pm 0.04$ & $770,221.21 \pm 37,331.73$ \\
\hline$(50,1,5) 57.38 \pm 5.01$ & $527,682.14 \pm 2,663.33$ & $0.66 \pm 0.06$ & $0.43 \pm 0.03$ & $812,531.72 \pm 47,958.66$ \\
\hline \multicolumn{5}{|c|}{ Dataset $270 \%$} \\
\hline$(50,0,1) 70.92 \pm 5.78$ & $902,040.57 \pm 4,206.39$ & $0.64 \pm 0.05$ & $0.45 \pm 0.03$ & $908,792.96 \pm 56,879.39$ \\
\hline$(50,1,0) 84.45 \pm 6.95$ & $674,078.31 \pm 6,146.74$ & $0.72 \pm 0.04$ & $0.34 \pm 0.03$ & $1,227,042.34 \pm 75,250.72$ \\
\hline$(50,1,1) 70.05 \pm 4.89$ & $902,380.79 \pm 3,655.72$ & $0.63 \pm 0.06$ & $0.44 \pm 0.03$ & $914,333.94 \pm 63,482.50$ \\
\hline$(50,1,2) 70.98 \pm 5.27$ & $902,769.29 \pm 3,141.76$ & $0.61 \pm 0.0$ & $0.45 \pm 0.03$ & $881,950.91 \pm 54,188.14$ \\
\hline$(50,1,5) 69.18 \pm 4.52$ & $901,764.91 \pm 3,425.91$ & $0.63 \pm 0.05$ & $0.45 \pm 0.03$ & $914,228.95 \pm 50,084.02$ \\
\hline
\end{tabular}


Table 8 Mann-Whitney-Wilcoxon test for dataset 1

\begin{tabular}{|c|c|c|c|}
\hline \multirow[b]{2}{*}{ ACS } & Optimal & \multirow[b]{2}{*}{$\mathrm{ACS}$} & \multirow[b]{3}{*}{ NSGA-II } \\
\hline & $\begin{array}{l}\mathrm{U}=145 n_{1}=19 n_{2}=14 \\
p_{\text {value }}=6.79 * 10^{-1}\end{array}$ & & \\
\hline NSGA-II & $\begin{array}{l}\mathrm{U}=134 n_{1}=19 n_{2}=18 \\
p_{\text {value }}=4.36 * 10^{-1}\end{array}$ & $\begin{array}{l}\mathrm{U}=88 n_{1}=14 n_{2}=12 \\
p_{\text {value }} 8.59 * 10^{-1}\end{array}$ & \\
\hline GRASP & $\begin{array}{l}\mathrm{U}=132.5 n_{1}=19 n_{2}=11 \\
p_{\text {value }}=2.33 * 10^{-1}\end{array}$ & $\begin{array}{l}\mathrm{U}=95 n_{1}=14 n_{2}=11 \\
p_{\text {value }}=3.4 * 10^{-1}\end{array}$ & $\begin{array}{l}\mathrm{U}=79.5 n_{1}=12 n_{2}=11 \\
p_{\text {value }}=4.13 * 10^{-1}\end{array}$ \\
\hline \multicolumn{4}{|c|}{$\begin{array}{l}\text { Results for the comparison of the Pareto-fronts obtained for dataset } 1 \\
\text { with a development effort bound of the } 30 \% \text { of the total software development resources }\end{array}$} \\
\hline & Optimal & & \\
\hline ACS & $\begin{array}{l}\mathrm{U}=268.4 n_{1}=27 n_{2}=18 \\
p_{\text {value }}=5.59 * 10^{-1}\end{array}$ & $\mathrm{ACS}$ & \\
\hline NSGA-II & $\begin{array}{l}\mathrm{U}=249 n_{1}=27 n_{2}=16 \\
p_{\text {value }}=4.19 * 10^{-1}\end{array}$ & $\begin{array}{l}\mathrm{U}=145.5 n_{1}=18 n_{2}=16 \\
p_{\text {value }}=9.95 * 10^{-1}\end{array}$ & NSGA-II \\
\hline GRASP & $\begin{array}{l}\mathrm{U}=280 n_{1}=27 n_{2}=18 \\
p_{\text {value }}=4.01 * 10^{-1}\end{array}$ & $\begin{array}{l}\mathrm{U}=170.5 n_{1}=18 n_{2}=18 \\
p_{\text {value }}=7.90 * 10^{-1}\end{array}$ & $\begin{array}{l}\mathrm{U}=154 n_{1}=16 n_{2}=18 \\
p_{\text {value }}=7.45 * 10^{-1}\end{array}$ \\
\hline
\end{tabular}

with a development effort bound of the $50 \%$ of the total software development resources

\begin{tabular}{|c|c|c|c|}
\hline & Optimal & & \\
\hline ACS & $\begin{array}{l}\mathrm{U}=391 n_{1}=32 n_{2}=21 \\
p_{\text {value }}=3.35 * 10^{-1}\end{array}$ & ACS & \\
\hline NSGA-II & $\begin{array}{l}\mathrm{U}=319,5 n_{1}=32 n_{2}=16 \\
p_{\text {value }}=3.25 * 10^{-1}\end{array}$ & $\begin{array}{l}\mathrm{U}=180 n_{1}=21 n_{2}=16 \\
p_{\text {value }}=7.28 * 10^{-1}\end{array}$ & NSGA-II \\
\hline GRASP & $\begin{array}{l}\mathrm{U}=440 n_{1}=32 n_{2}=23 \\
p_{\text {value }}=2.25 * 10^{-1}\end{array}$ & $\begin{array}{l}\mathrm{U}=253.5 n_{1}=23 n_{2}=21 \\
p_{\text {value }}=7.79 * 10^{-1}\end{array}$ & $\begin{array}{l}\mathrm{U}=196.5 n_{1}=23 n_{2}=16 \\
p_{\text {value }}=7.24 * 10^{-1}\end{array}$ \\
\hline
\end{tabular}
with a development effort bound of the $70 \%$ of the total software development resources

\begin{tabular}{l|l}
\hline How explain the & \\
test, When... & The difference between the two samples is highly significant \\
$P<0.001$ & Two samples are significantly different \\
$P<0.01$ & The difference between the two samples is marginally significant \\
$P<0.05$ & Two samples are not significantly different \\
$P>=0.05$ & \\
\hline
\end{tabular}


Table 9 Mann-Whitney-Wilcoxon test for dataset 2

\begin{tabular}{|c|c|c|}
\hline NSGA-II & $\begin{array}{c}\text { ACS } \\
=2560.5, n_{1}=78, n_{2}=57 \\
p_{\text {value }}=1.29 * 10^{-1}\end{array}$ & NSGA-II \\
\hline GRASP & $\begin{array}{c}U=2505, n_{1}=72, n_{2}=57 \\
p_{\text {value }}=2.94 * 10^{-2}\end{array}$ & $\begin{array}{l}U=3792, n_{1}=78, n_{2}=72 \\
p_{\text {value }}=1.3 * 10^{-4}\end{array}$ \\
\hline \multicolumn{3}{|c|}{$\begin{array}{l}\text { Results for the Pareto-fronts obtained for dataset } 2 \\
\text { with a development effort bound of } 30 \%\end{array}$} \\
\hline NSGA-II & $\begin{array}{c}\mathrm{ACS} \\
U=4122.5, n_{1}=86, n_{2}=76 \\
p_{\text {value }}=3.6 * 10^{-3}\end{array}$ & NSGA-II \\
\hline GRASP & $\begin{array}{c}U=3953, n_{1}=92, n_{2}=76 \\
p_{\text {value }}=1.42 * 10^{-1}\end{array}$ & $\begin{array}{c}U=4089.5, n_{1}=92, n_{2}=86 \\
p_{\text {value }}=6.96 * 10^{-1}\end{array}$ \\
\hline \multicolumn{3}{|c|}{$\begin{array}{l}\text { Results for the Pareto-fronts obtained for dataset } 2 \\
\text { with a development effort bound of } 50 \%\end{array}$} \\
\hline NSGA-II & $\begin{array}{c}\mathrm{ACS} \\
\begin{array}{c}U=4965, n_{1}=96, n_{2}=79 \\
p_{\text {value }}=3.48 * 10^{-4}\end{array}\end{array}$ & NSGA-II \\
\hline GRASP & $\begin{aligned} U= & 7060.5, n_{1}=144, n_{2}=79 \\
& p_{\text {value }}=2.62 * 10^{-3}\end{aligned}$ & $\begin{aligned} U= & 7063.5, n_{1}=144, n_{2}=96 \\
& p_{\text {value }}=7.71 * 10^{-1}\end{aligned}$ \\
\hline \multicolumn{3}{|c|}{$\begin{array}{c}\text { Results for the Pareto-fronts obtained for dataset } 2 \\
\text { with a development effort bound of } 70 \%\end{array}$} \\
\hline $\begin{array}{l}\text { When ... } \\
P<0.001 \\
P<0.01 \\
P<0.05 \\
P>=0.05\end{array}$ & \multicolumn{2}{|c|}{$\begin{array}{l}\text { The difference between the two samples is highly significant } \\
\text { Two samples are significantly different } \\
\text { The difference between the two samples is marginally significant } \\
\text { Two samples are not significantly different }\end{array}$} \\
\hline
\end{tabular}

